\title{
Targetable cellular signaling events mediate vascular pathology in vascular Ehlers-Danlos syndrome
}

\author{
Caitlin J. Bowen, ${ }^{1,2}$ Juan Francisco Calderón Giadrosic,, ${ }^{1}$ achary Burger, ${ }^{1}$ Graham Rykiel, ${ }^{1}$ Elaine C. Davis, ${ }^{3}$ Mark R. Helmers, ${ }^{1}$ \\ Kelly Benke, ${ }^{4}$ Elena Gallo MacFarlane, ${ }^{1}$ and Harry C. Dietz ${ }^{1,2}$ \\ IInstitute of Cenetic Medicine, Johns Hopkins University School of Medicine, Baltimore, Maryland, USA. ${ }^{2}$ Howard Hughes Medical Institute, Chevy Chase, Maryland, USA. ${ }^{3}$ Anatomy and Cell Biology, \\ McGill University, Montreal, Quebec, Canada. ${ }^{4}$ Department of Mental Health, Johns Hopkins Bloomberg School of Public Health, Baltimore, Maryland, USA.
}

\begin{abstract}
Vascular Ehlers-Danlos syndrome (vEDS) is an autosomal-dominant connective tissue disorder caused by heterozygous mutations in the COL3A1 gene, which encodes the pro- $\alpha 1$ chain of collagen III. Loss of structural integrity of the extracellular matrix is believed to drive the signs and symptoms of this condition, including spontaneous arterial dissection and/or rupture, the major cause of mortality. We created 2 mouse models of vEDS that carry heterozygous mutations in Col3a1 that encode glycine substitutions analogous to those found in patients, and we showed that signaling abnormalities in the PLC/IP ${ }_{3} /$ PKC/ERK pathway (phospholipase C/inositol 1,4,5-triphosphate/protein kinase C/extracellular signal-regulated kinase) are major mediators of vascular pathology. Treatment with pharmacologic inhibitors of ERK1/2 or PKC $\beta$ prevented death due to spontaneous aortic rupture. Additionally, we found that pregnancy- and puberty-associated accentuation of vascular risk, also seen in vEDS patients, was rescued by attenuation of oxytocin and androgen signaling, respectively. Taken together, our results provide evidence that targetable signaling abnormalities contribute to the pathogenesis of vEDS, highlighting unanticipated therapeutic opportunities.
\end{abstract}

\section{Introduction}

Vascular Ehlers-Danlos syndrome (vEDS) (OMIM 130050) is a rare genetic connective tissue disorder. Patients with vEDS present with thin, translucent skin, easy bruising, and a characteristic facial appearance. Most critically, they are at risk for spontaneous rupture of the major arteries, hollow organs, and gravid uterus (1-4). In contrast to other heritable vasculopathies, such as Marfan syndrome (MFS) and Loeys-Dietz syndrome (LDS), dissection in VEDS often occurs without prior aneurysm, and rupture can involve any muscular artery (5-8). These features make disease prediction and monitoring difficult, and the presenting sign in the majority of adults diagnosed with vEDS is catastrophic vascular dissection or organ rupture, with $25 \%$ of patients experiencing a major complication by age 20 years (1-3).

vEDS is caused by autosomal dominant mutations in COL $3 \mathrm{~A} 1$, which encodes the pro- $\alpha 1$ chain of collagen III, a major structural matrix element in blood vessels and hollow organs (1). Mutations that result in production of abnormal protein, most typically glycine substitutions in the repetitive Gly-X-Y sequence required for triple helix formation or splice site mutations, are associated with a more severe phenotype than are mutations that result in simple haploinsufficiency. The onset and severity of disease manifestation is highly variable even within families. Contributors include sexual dimorphism, with greater severity in males, and potent-

Conflict of interest: CJB, JFCG, and HCD have submitted patent application PCT/ US19/056616 for pharmacological treatment of vascular Ehlers-Danlos syndrome. Copyright: () 2020, American Society for Clinical Investigation.

Submitted: June 4, 2019; Accepted: October 15, 2019; Published: December 17, 2019. Reference information: / Clin Invest. 2020;130(2):686-698.

https://doi.org/10.1172/JCl130730. ially other sources of genetic or environmental modification that remain to be defined (9-12).

The current understanding of this condition is that reduced amounts of collagen III cause loss of structural integrity of the tissues, which directly leads to the tissue fragility and failure in vEDS $(3,11,13)$. Current clinical management, which consists of treatments aimed at reducing blood pressure and aortic wall stress, reflects this view (13). Surgical management of vascular disease is avoided until impending catastrophe due to operative complications associated with significant morbidity and mortality (3). Little is known about the precise functional consequences of collagen III deficiency in the extracellular matrix, or of the effects that alterations in the extracellular matrix have on intracellular biological processes in this condition, including the potential for nonproductive compensatory responses by neighboring cells that might contribute to disease pathogenesis $(4,14-17)$. It is currently unknown if signaling pathways found to be altered in other vascular genetic disorders, such as such as MFS and LDS (18-20), are also involved in vEDS disease pathogenesis.

Preclinical investigation of vEDS has been severely restricted by the lack of animal models that recapitulate the vascular pathology or molecular mechanism of disease. Previous Col3a1 haploinsufficient models have a normal life expectancy and do not show any signs of vascular disease, whereas the complete null state results in death within 48 hours of birth (21), preventing further analysis. More recent transgenic mouse models that overexpress Col3a1 ${ }^{\text {1182S/+ }}$ die from severe dermal wounds before presenting with vascular complications (16). Another mouse model in which Col3a1 is inactivated by a large $185-\mathrm{kb}$ deletion encompassing the promoter region and first 39 exons of the Col3a1 gene, presents with aortic rupture at 4-10 weeks of age (4). Another study proposes that this 
mouse model harbors an internal deletion that could give rise to a mutant protein, but this was not demonstrated directly $(4,22)$.

In this study, we generate and characterize 2 knockin vEDS mouse models which harbor heterozygous glycine substitutions previously observed in people with vEDS and result in spontaneous vascular rupture and death. Comprehensive transcriptome profiling of the descending thoracic aorta in vEDS mice revealed altered cellular signaling events, which when attenuated pharmacologically resulted in overt phenotypic rescue.

\section{Results}

Col3a1 $1^{\mathrm{G} 209 \mathrm{~S} /+}$ and Col3a1 ${ }^{\mathrm{G} 938 \mathrm{D} /{ }_{+}}$mice recapitulate $v E D S$ phenotypes. In order to investigate the mechanisms of aortic rupture in vEDS, we used CRISPR/Cas9 (23) to create 2 mouse models of vEDS. Separate heterozygous glycine substitutions were introduced at the beginning or end of the triple helical collagenous domain: glycine to serine at codon 209 (Col3a1 $\left.{ }^{\mathrm{G} 209 \mathrm{~S} /+}\right)$ or glycine to aspartic acid at codon 938 (Col3a1 ${ }^{\text {G938D/+})}$ (Figure 1, A and B, and Supplemental Table 1; supplemental material available online with this article; https://doi.org/10.1172/JCI130730DS1). Both models recapitulate vEDS vascular phenotypes with sudden death due to aortic rupture (Supplemental Figure 1 and Figure 1, C and D). Among 100 vEDS mice examined after acute death, 90 showed hemothorax and the remaining 10 showed hemoperitoneum. Notably absent was any occurrence of hemopericardium, a common finding in mouse models predisposed to ascending aortic disease. While isolated aortic rupture was the most common cause of death in our mouse models, there was occasional accompanying aortic dissection which always presented in the proximal descending thoracic aorta (Supplemental Figure 1). In 12 of 18 mice injected with latex in the left ventricle after presenting with hemothorax, we visualized latex exiting the aorta at the level of the descending thoracic aorta, indicating rupture at that level. For the remaining 6 mice, extravasation occurred in the distal transverse arch at the level of the left subclavian artery. As predicted by the fact that collagen III assembly progresses from the C- to N-terminus (24), Col3a $1^{\mathrm{G} 938 \mathrm{D} /+}$ mice display a more severe phenotype, with a median survival of

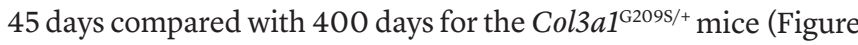
$1, \mathrm{C}$ and D). Consistent with what is observed in patients, neither mouse model shows a tendency for formation of aortic root or ascending aortic aneurysm, although $\mathrm{Col} 3 \mathrm{a} 1^{\mathrm{G} 938 \mathrm{D} /+}$ mice appear to have smaller aortas, likely attributable to their smaller body size (ref. 25 and Supplemental Figure 2).

Although the aortic wall architecture is relatively preserved in both models, minor alterations include occasional elastic fiber breaks, decreased aortic wall thickness, and decreased collagen content at 2 months of age (Figure 1, E-H). Analysis by transmission electron microscopy shows disruption of elastic lamellar units, including thickened elastic fibers with a moth-eaten appearance, disarray of vascular smooth muscle cells (VSMCs) between fibers, and a paucity of collagen fibrils that normally occupy the intervening space between VSMCs and adjacent elastic fibers (Supplemental Figure 3). Collagen fibrils within the aortic media showed wide variation in diameter with a generally smaller size when compared with control mice (Supplemental Figure 3), consistent with previous reports $(4,16,26)$. Fibroblasts within the aortic adventitia of vEDS mice showed gross distension of the endoplasmic reticulum
(ER), presumably due to impaired trafficking of abnormally folded collagen III (Supplemental Figure 3), also consistent with previous observations in patient-derived fibroblasts (26).

Blood pressure reduction is ineffective in improving survival of $v E D S$ mouse models. In order to evaluate the effect of blood pressure reduction on prevention of vascular rupture in vEDS mouse models, we assessed the effects of losartan, propranolol, atenolol, and amlodipine, all drugs previously tested in MFS and LDS mouse models and known to reduce systemic blood pressure in mice $(6,8,18-20,27)$. Despite all of these drugs causing the predicted reduction in blood pressure (Supplemental Figure 4), no treatment resulted in increased survival, with losartan, propranolol, and atenolol having no impact, and amlodipine trending toward an increased risk of aortic rupture (Supplemental Figure 4).

We also tested the effect of celiprolol, a $\beta 1$ antagonist $/ \beta 2$ agonist that previous work has proposed, on the basis of a small trial, to delay adverse events in patients with vEDS $(13,28)$. Surprisingly, celiprolol accelerated rather than reduced death from aortic rupture in both the severe Col3a1 ${ }^{\mathrm{G} 938 \mathrm{D} /+}$ and mild Col3a1 $1^{\mathrm{G} 209 \mathrm{~S} /+}$ vEDS mouse models, despite having the predicted effect on pulse rate (Supplemental Figure 4).

$v E D S$ aortas display a molecular signature for excessive $P L C / I P_{3} /$ $P K C / E R K$ signaling. In order to elucidate which signaling abnormalities, if any, mediate disease pathology in vEDS, we performed comparative transcriptional profiling by high-throughput RNA sequencing (RNA-seq) on the isolated proximal descending thoracic aortas of 10 -week-old Col3a1 $1^{\mathrm{G} 938 \mathrm{D} /+}$ and $\mathrm{Col} 3 \mathrm{a} 1^{+/+}$mice. This region of the aorta is where dissection and/or rupture most commonly occurs in our vEDS mouse models (Supplemental Figure 5).

We identified 170 consistently differentially expressed transcripts (probability of differential expression >0.95) between Col3a1 ${ }^{\mathrm{G} 938 \mathrm{D} /+}$ aortas compared with $\mathrm{Col3a1}^{+/+}$aortas (Figure 2A). Network analysis indicated elevated mitogen-activated protein kinase (MAPK) activity, including p38, c-Jun N-terminal kinases (JNK), Akt, and extracellular signal-regulated kinases (ERK1/2) (Supplemental Figure 6). Furthermore, upstream and gene-set enrichment analyses suggested that transcriptional differences in vEDS aortas may be driven by excessive activity of ERK and $\mathrm{Ca}^{++}$/ $\mathrm{G}_{\alpha \mathrm{q}}$ protein coupled receptors (GPCRs), which signal through the $\mathrm{PLC} / \mathrm{IP}_{3} / \mathrm{PKC} / \mathrm{ERK}$ axis (Figure $2 \mathrm{~B}$ and Supplemental Figure 7).

In order to validate the predictions of transcriptome profiling, we analyzed aortic lysates derived from control, Col3a $1^{\mathrm{G} 938 \mathrm{D} /+}$, and Col3a1 $1^{\mathrm{G} 209 \mathrm{~S} /+}$ mice at 8 weeks of age by immunoblots, using antibodies directed against phosphorylated ERK1/2 and PKC $\beta$. We found that levels of ERK1/2 and PKC $\beta$ phosphorylation were significantly increased in the aorta of both Col3a1 $1^{\mathrm{G} 938 \mathrm{D} /+}$ and Col3a1 $1^{\mathrm{G} 209 \mathrm{~S} /+}$ mice, suggesting that these pathways might be involved in disease pathogenesis (Figure 2, C-E).

Attenuation of $\mathrm{PLC} / \mathrm{IP}_{3} / \mathrm{PKC} / \mathrm{ERK}$ signaling prevents death due to aortic rupture. In view of the beneficial effects of PLC/IP $/$ PKC/ERK axis inhibition in mouse models of MFS (19), and to determine if the increased activation of PKC and/or ERK was a cause or a consequence of disease, we then tested if attenuation of this signaling pathway would decrease the risk of aortic rupture in Col3a1 $1^{\mathrm{G} 938 \mathrm{D} /+}$ mice. Treatment of Col3a1 ${ }^{\mathrm{G} 938 \mathrm{D} /+}$ mice with ruboxistaurin, an orally administered pharmacologic agent that specifically inhibits PKC $\beta$ (29), resulted in 94\% survival after 45 
A

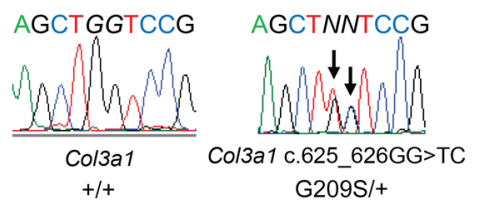

B
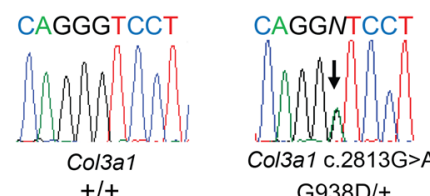

G938D/+
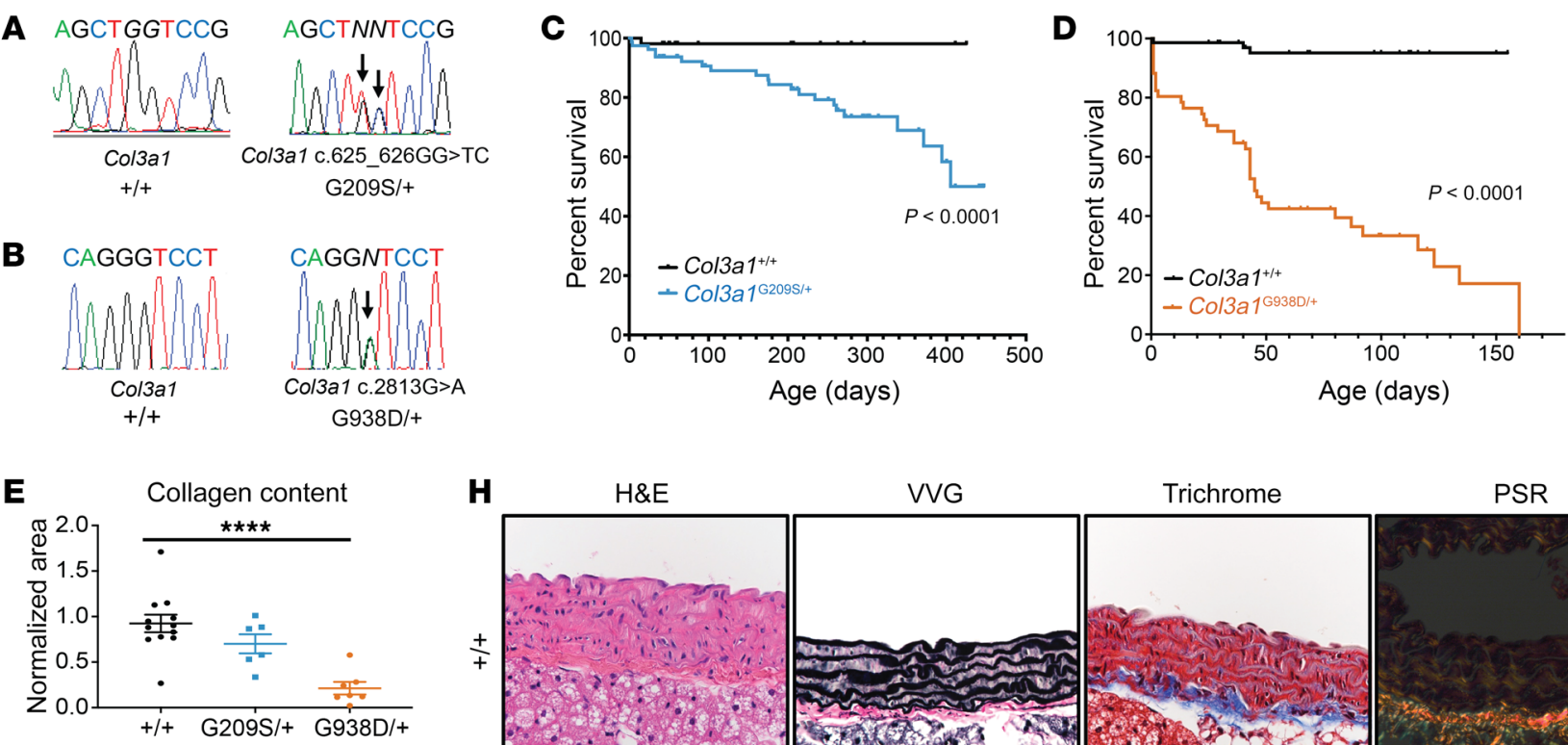

$\mathbf{F}$

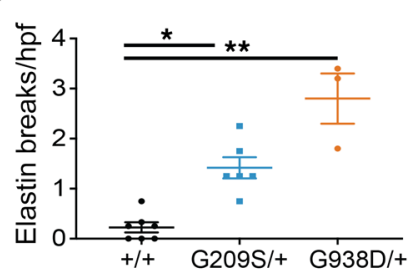

H

H\&E

VVG Trichrome PSR

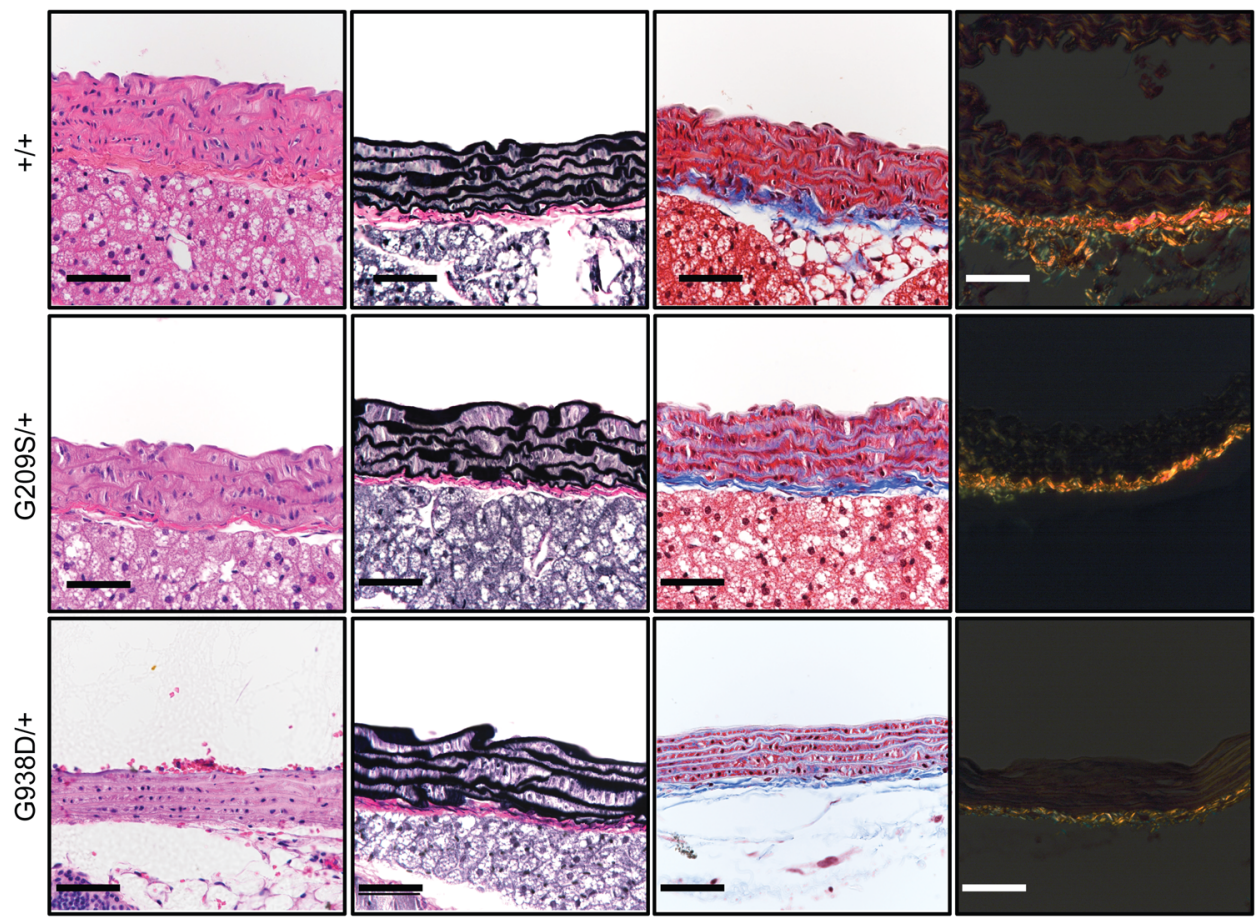

G

Aortic wall thickness

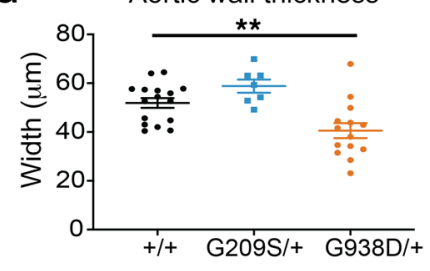

Figure 1. Col3a1 ${ }^{\mathrm{C2095/+}}$ and Col3a1 ${ }^{\mathrm{Gg} 38 \mathrm{~d} /+}$ mice recapitulate vEDS phenotypes. (A) Sanger sequencing of genomic DNA confirmed the intended Col3a1 c.625_626CG>TC corresponding to G209S. (B) Sanger sequencing of genomic DNA confirmed the intended Col3a1 c.2813G>A corresponding to G938D. (C)

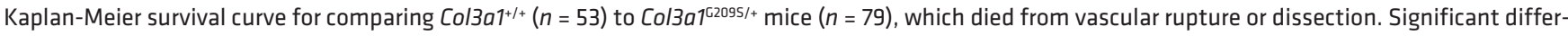
ences were calculated using log-rank (Mantel-Cox) analysis. (D) Kaplan-Meier survival curve for comparing Col3a1+/+ $(n=78)$ to Col3a ${ }^{\mathrm{Cg380} /+}$ mice $(n=51)$, which died from vascular rupture or dissection. Significant differences were calculated using log-rank (Mantel-Cox) analysis. (E) Quantification of collagen content in aortic cross sections, as measured by normalized PSR intensity. Error bars show mean $\pm \mathrm{SEM}$. Asterisks signify significant differences using 1-way ANOVA with Dunnett's multiple comparisons post hoc test. ${ }^{* * *} P<0.0001$, DF $=2, F=13.97$. (F) Quantification of elastin breaks in VVG-stained aortic cross sections. Error bars show mean \pm SEM. Asterisks signify significant differences using Kruskal-Wallis with Dunn's multiple comparisons post hoc test. ${ }^{*} P<0.05$, ${ }^{* *} P<0.01$. (C) Quantification of aortic wall thickness in aortic cross sections. Error bars show mean \pm SEM. Asterisks signify significant differences using 1-way ANOVA with Dunnett's multiple comparisons post hoc test. ${ }^{* *} P<0.01, \mathrm{DF}=2, \mathrm{~F}=10.16$. (H) Histological staining (H\&E $=$ hematoxylin \& eosin, VVG = Verhoeff Van Gieson, Masson's Trichrome, and PSR = Picrosirius Red) of wild-type and vEDS aortic cross sections. Scale bars: $50 \mu \mathrm{m}$.

days of treatment, compared with only $52 \%$ survival with no treatment (Figure 3A). This effect was associated with the expected inhibition of $\mathrm{PKC} \beta$ autophosphorylation as well as with reduction of phosphorylation of ERK1/2 in the aortic wall, as assessed by immunoblots of aortic lysates (Figure 3B).

These data suggested that PKC-dependent ERK activation may be a critical driver of aortic disease in vEDS. In order to directly test the relevance of ERK activation, we next treated Col3a1 $1^{\mathrm{G} 938 \mathrm{D} /+}$ mice with cobimetinib, an FDA-approved inhibitor of MEK, the kinase that activates ERK (30). This treatment resulted in $90 \%$ survival after 45 days of treatment, compared with only $52 \%$ survival with no treatment (Figure $3 \mathrm{C}$ ). Immunoblot of aortic lysates showed that increased survival correlated with the expected reduction in phosphorylation of ERK1/2, the downstream substrate for MEK, as well as reduced $\mathrm{PKC} \beta$ phosphorylation (Figure $3 \mathrm{~B})$. Neither cobimetinib nor ruboxistaurin had an effect on blood pressure or on aortic wall morphology or collagen content in vEDS mice (Supplemental Figure 8).

In order to test if other FDA-approved medications that target the same pathway afford similar protection, we next treat- 

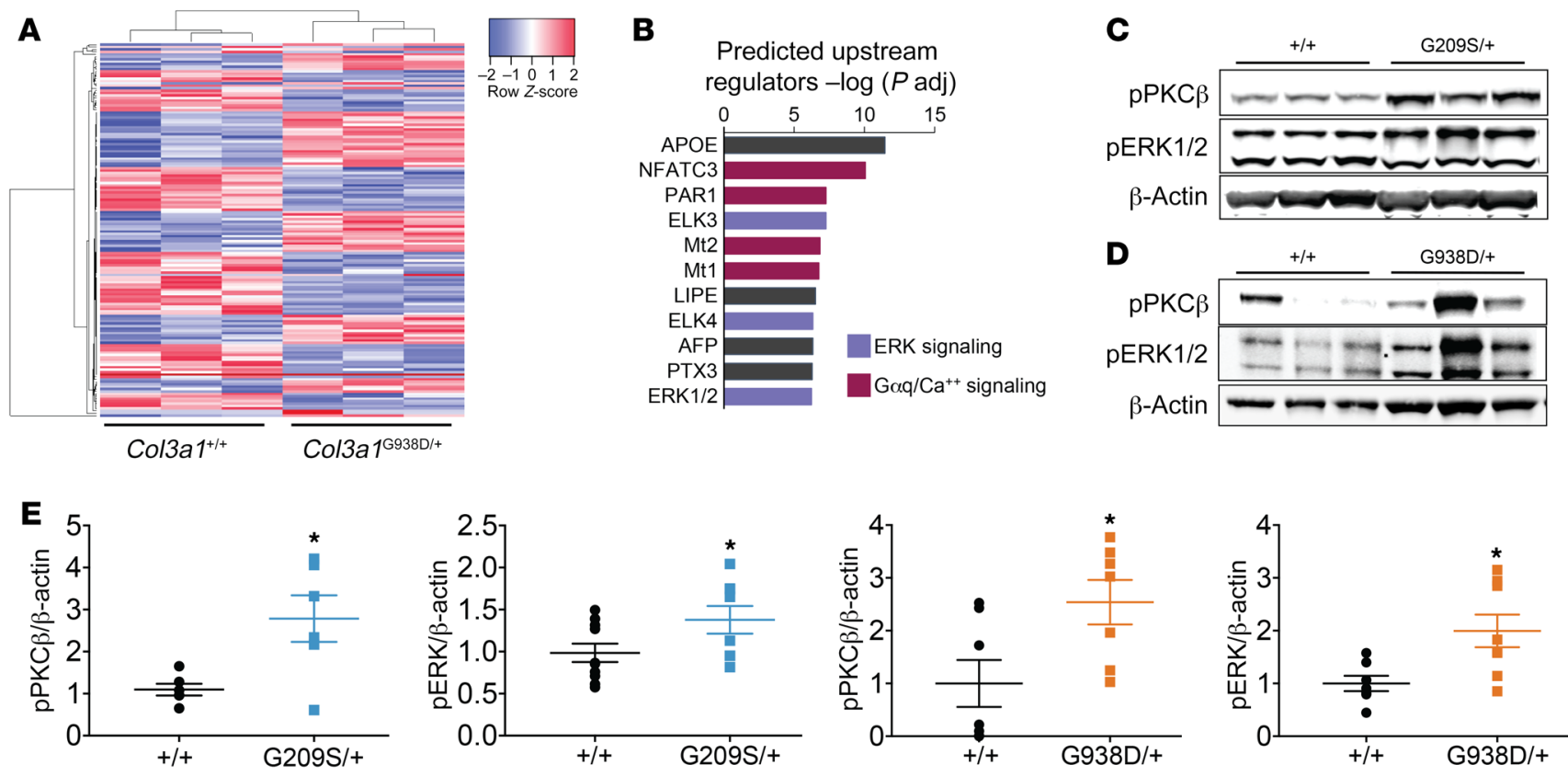

Figure 2. vEDS aortas display a molecular signature for excessive PKC/ERK signaling. (A) Unsupervised hierarchical clustering using the most differentially expressed genes from RNAseq was performed, and vEDS samples clustered separately from controls. (B) Upstream analysis based on differentially expressed genes. Significant enrichment was determined using Fisher's exact test. (C) Representative Western blot analysis of pPKC $\beta$ and pERK compar-

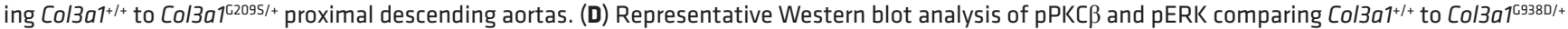
proximal descending aortas. (E) Quantification of pPKC $\beta$ and pERK levels normalized to $\beta$-actin loading control comparing Col3a ${ }^{1^{++}}(n=6)$ to $\mathrm{Col} 3 a 7^{\mathrm{C} 2095 /+}$ $(n=6)$ and Col3a ${ }^{\mathrm{G9380/+}}(n=8)$ aortas. Error bars show mean \pm SEM. Asterisks signify significant differences using 2 -tailed Student's $t$ test (pERK/C209S T $\left.=2.053, \mathrm{DF}=16 ; \mathrm{pPKC} \beta / \mathrm{C} 209 \mathrm{~S} \mathrm{~T}=2.950, \mathrm{DF}=10 ; \mathrm{pERK} / \mathrm{G} 938 \mathrm{D} \mathrm{T}=2.770, \mathrm{DF}=13 ;{ }^{*} P<0.05\right)$ or Mann-Whitney test $\left(\mathrm{C} 938 \mathrm{D} / \mathrm{pPKC} \beta ;{ }^{*} P<0.05\right)$ depending on Shapiro-Wilk normality tests.

ed Col3a1 ${ }^{\mathrm{G} 38 \mathrm{D} /+}$ mice with hyidralazine. Hydralazine is a blood pressure medication that works, at least in part, by blocking $\mathrm{IP}_{3}$ mediated calcium release from the ER and hence PKC $\beta$ activation (31). When initiating treatment at birth, we observed significant improvement in survival, with $97 \%$ of treated mice surviving to 45 days - the median age of survival for an untreated Col3a $1^{\mathrm{G93} 3 \mathrm{D} /+}$ mouse (Figure 4A). Protection from aortic rupture in Col3a1 ${ }^{\mathrm{G} 938 \mathrm{D} /+}$ mice treated with hydralazine was abruptly lost around the time of sexual maturity ( 50 days). While this phenomenon was observed in both sexes, the effect was greatly exaggerated in male $\mathrm{Col} 3 \mathrm{a} 1^{\mathrm{G} 938 \mathrm{D} /+}$ mice $(25 \%$ vs. $60 \%$ survival at 100 days for male and female Col3a $1^{\mathrm{G} 938 \mathrm{D} /+}$ mice, respectively), suggesting a potential role for androgens (Figure 4, B and C). In keeping with this hypothesis, we found that additional administration of the androgen receptor antagonist bicalutamide to mice receiving hydralazine led to $90 \%$ survival at 100 days of age in male mice and $100 \%$ survival in female mice (Figure 4, B and C). In contrast, treatment with bicalutamide alone attenuated the risk of vascular rupture (70\% survival after 60 days of treatment vs. 50\% survival in untreated Col3a $1^{\mathrm{G938D} /+}$ mice) but did not completely prevent rupture (Figure 4D). Surprisingly, male mice on hydralazine continued to be protected from vascular rupture upon removal of bicalutamide after puberty, suggesting a time-dependence for the deleterious effects of androgen signaling (Figure 4C). Similar to what we observed with bicalutamide, concomitant treatment with hydralazine and another FDA-approved competitive antagonist of the androgen receptor, spironolactone, resulted in $100 \%$ survival of Col3a $1^{\mathrm{G} 938 \mathrm{D} /+}$ mice of both sexes at the end of the trial 65 days later, as compared with $43 \%$ survival in untreated animals (Figure $4 \mathrm{E})$. Survival correlated with the status of $P K C \beta$ and ERK1/2 phosphorylation in the aortic wall, which was increased in Col3a1 ${ }^{\mathrm{G} 338 \mathrm{D} /+}$ mice, reduced in Col3a1 ${ }^{6388 D /+}$ mice prior to sexual maturity upon treatment with hydralazine, increased in $\mathrm{Col} 3 a 1^{\mathrm{G} 938 \mathrm{D} /+}$ mice after sexual maturity despite ongoing treatment with hydralazine, but again reduced in sexually mature Col3a1 ${ }^{\mathrm{G} 938 \mathrm{D} / /}$ mice treated with both hydralazine and spironolactone (Figure 4, F and G, Supplemental Figure 9). There was no difference in androgen receptor expression in the aorta between $\mathrm{Col}_{3} \mathrm{al}^{+/+}$and Col3a $\mathrm{1}^{\mathrm{G} 38 \mathrm{D} /+}$ mice (Supplemental Figure 9), suggesting that physiologically normal levels of androgen signaling are cross-talking with the elevated $\mathrm{PLC} / \mathrm{IP}_{3} / \mathrm{PKC} / \mathrm{ERK}$ signaling cascade. We did not observe differences between male and female survival for any other trial (Supplemental Table 2).

Oxytocin antagonism prevents pregnancy-associated aortic dissection. Pregnancy-associated aortic dissection is the most common cause of death in women with vEDS of child-bearing age (1, $11,32,33)$. Although increased risk of vascular events in pregnancy has historically been attributed to hemodynamic stress, we have previously shown that pregnancy-associated aortic dissection in MFS mice is largely driven by lactation-associated oxytocin release and oxytocin-induced PLC/IP 3 /PKC/ERK signaling (34). In view of these data, we hypothesized that pregnancy-associated vascular events in vEDS are driven by overactivation of PLC/IP $/$ PKC/ERK by a similar mechanism. 
A

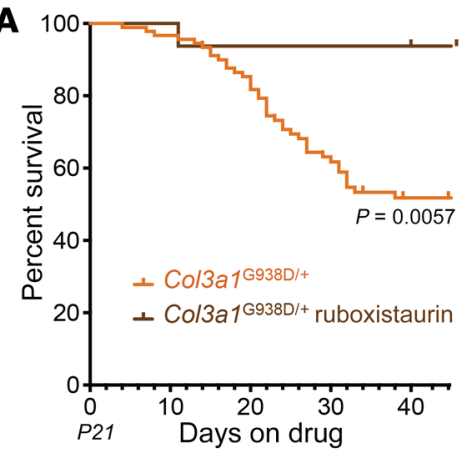

C

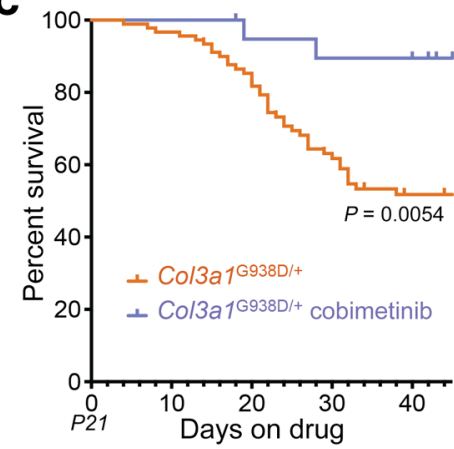

B
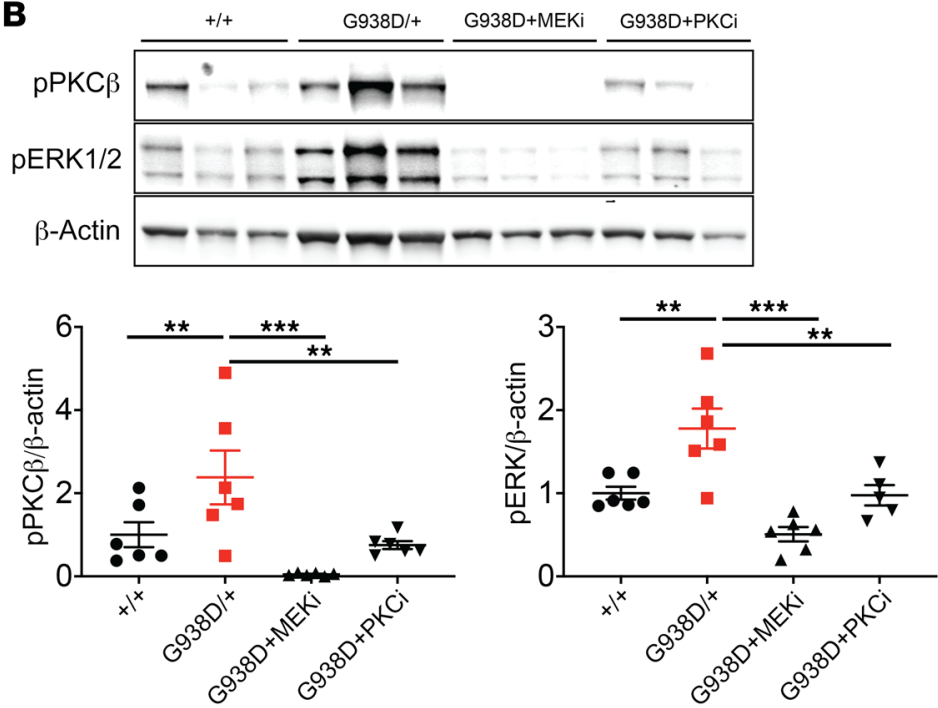

Figure 3. Inhibition of excessive PKC $\beta$ or ERK signaling prevents death due to aortic dissection. (A) Kaplan-Meier survival curve comparing Col3a19938D/+ $(n=93)$ to Col3a1 ${ }^{\mathrm{G938D/+}}(n=16)$ mice receiving ruboxistaurin in the diet starting at weaning and continuing for 40 days. Significant differences were calculated using log-rank (Mantel-Cox) analysis. P21 = postnatal day 21. (B) Representative Western blot and analysis of pPKC $\beta$ and pERK comparing Col3a1+/+ $(n=6)$ to Col3a1 ${ }^{6938 D /+}(n=6)$ proximal descending aortas and quantification of pPKC $\beta$ and pERK levels normalized to $\beta$-actin loading control for vEDS aortas. Error bars show mean \pm SEM. Asterisks signify significant differences using 1-way ANOVA with Dunnett's multiple comparisons post hoc test.

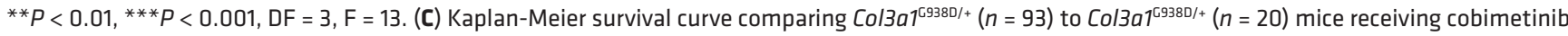
in the drinking water starting at weaning and continuing for 40 days. Significant differences were calculated using log-rank (Mantel-Cox) analysis. P21 = postnatal day 21. All findings from drug trials are based on analyses using a universal control group with $n=93$ across all drug tests that started at P21. FDR-adjusted $P$ values are presented in Supplemental Table 3.

In order to test this hypothesis, we examined the effect of pregnancy and lactation on the survival of Col3a1 $1^{\mathrm{G} 209 \mathrm{~S} /+}$ mice, which have a milder phenotype and reach reproductive age more frequently. In this mouse model, we found that pregnancy and lactation is associated with $54 \%$ lethality due to arterial rupture in the first 30 days postpartum, compared with $96 \%$ survival during this same time period in never-pregnant Col3a1 ${ }^{\mathrm{G} 209 \mathrm{~S} /+}$ females (Figure 5A). To determine whether lactation played a role in pregnancy-associated aortic rupture, we removed Col3a1 $1^{\mathrm{G} 209 \mathrm{~s} /+}$ mothers from their pups right after birth, effectively preventing lactation-induced oxytocin release in the postpartum period. This simple manipulation resulted in $100 \%$ survival of Col3a $1^{\mathrm{G} 209 \mathrm{~S} /+}$ mice in the postpartum period (Figure 5B). Moreover, treatment with a specific oxytocin receptor antagonist (35) led to enhanced postpartum survival ( 90\%) (Figure 5C), directly implicating oxytocin signaling in pregnancy-associated accentuation of vascular risk in Col3a1 ${ }^{\mathrm{G} 209 \mathrm{~S} /+}$ mice.

In order to test if postpartum elevation of PKC/ERK activation was driving mortality, we next tested the effect of inhibitors of MEK/ERK and IP3/PKC activation in postpartum lactating female mice. Initiation of either MEK/ERK inhibition using trametinib or $\mathrm{IP}_{3} /$ PKC inhibition using hydralazine in Col3a1 $1^{\mathrm{G} 209 \mathrm{~s} /+}$ mice at the time of delivery resulted in $95 \%$ survival at day 30 postpartum, compared with $46 \%$ survival in untreated lactating
Col3a1 ${ }^{\mathrm{G} 209 \mathrm{~S} /{ }^{+}}$mice (Figure 5, D and E). Analysis of ERK1/2 signaling in aortic lysates by immunoblotting and by analysis of expression of ERK target genes showed that activation of this pathway is increased in the aortas of never-pregnant $\mathrm{Col} 3 \mathrm{a} 1^{\mathrm{G} 209 \mathrm{~S} /+}$ females as compared with never-pregnant $\mathrm{Col}_{3 \mathrm{al}^{+/+}}$females, and that pregnancy and lactation cause significant further upregulation (Figure 6, A-D). Treatments that improved survival, including pup removal and treatment with hydralazine, trametinib, or an oxytocin receptor antagonist, also resulted in decreased ERK activation (Figure 6, A-D). Similar to what we observed in nonpregnant Col3a1 $1^{\mathrm{G} 938 \mathrm{D} /+}$ mice (Supplemental Figure 4), treatment with propranolol had no impact on survival in the postpartum period (Figure 5F), despite the expected reduction in blood pressure and heart rate (Supplemental Figure 4). Taken together, these data further support the notion that excessive activation of the PLC/ $\mathrm{IP}_{3} / \mathrm{PKC} / \mathrm{ERK}$ signaling pathway drives aortic rupture in both pregnant and nonpregnant vEDS mice.

\section{Discussion}

In this study, we create and describe 2 mouse models, Col3a1 $1^{\mathrm{G} 209 \mathrm{~s} /+}$ and Col3a1 ${ }^{\mathrm{G} 938 \mathrm{D} /+}$, that carry knockin mutations analogous to those known to cause vEDS in people and that faithfully recapitulate the main cause of death in this condition, premature death from spontaneous aortic rupture. This allowed investigation of disease patho- 

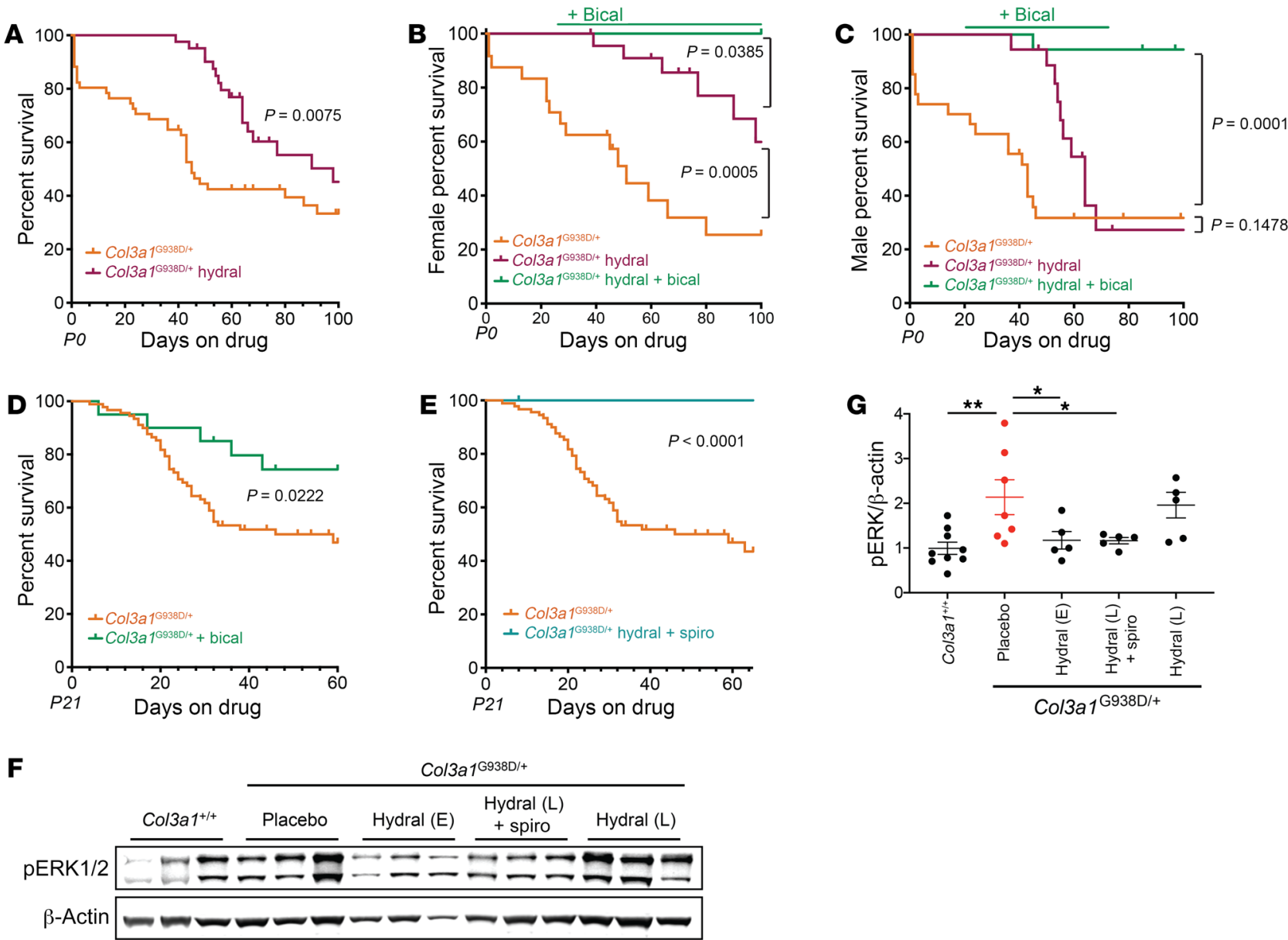

Figure 4. Androgen signaling at puberty increases the risk of aortic rupture. (A) Kaplan-Meier survival curve comparing Col3a ${ }^{69380 /+}(n=51)$ to $\mathrm{Col} 3 a 1^{69380 /+}$ $(n=41)$ mice receiving hydralazine from birth. (B) Kaplan-Meier survival curve comparing female Col3a $7^{\mathrm{Ca380/+}}(n=24)$ to female Col3a ${ }^{\mathrm{C9380} /+}(n=23)$ mice receiving hydralazine starting from birth and bicalutamide starting from weaning $(n=9)$. $P$ values shown are between untreated mice and mice receiving hydralazine (lower) and mice receiving hydralazine and mice receiving hydralazine and bicalutamide (upper). (C) Kaplan-Meier survival curve comparing male

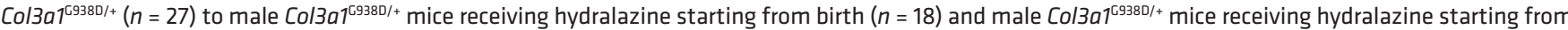
birth and bicalutamide starting from weaning and continuing for 2 months $(n=18)$. $P$ values shown are between untreated mice and mice receiving hydralazine (lower) and mice receiving hydralazine and mice receiving hydralazine and bicalutamide (upper). (D) Kaplan-Meier survival curve comparing Col3a19938/+

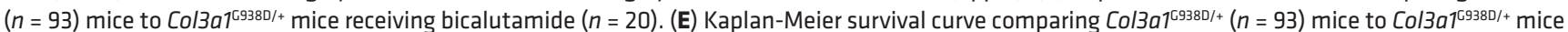
receiving hydralazine and spironolactone $(n=16)$. (F) Representative Western blot analysis of pERK comparing Col3a ${ }^{1^{++}}(n=9)$ to Col3a ${ }^{69380 /+}(n=7)$ mice, Col3a $7^{\mathrm{G9380/+}}$ mice on hydralazine sampled at age P40 (Hydral $(\mathrm{E}), n=5$ ), Col3a $7^{\mathrm{G9380/+}}$ mice on hydralazine and spironolactone sampled at age P70 (Hydral $(\mathrm{L})+$ spiro, $n=5$ ), and Col3a ${ }^{69380 /+}$ mice on hydralazine sampled at age P70 (Hydral $(\mathrm{L}), n=5$ ) proximal descending aortas. (C) Quantification of pERK levels normalized to $\beta$-actin-loading control for aortas. Error bars show mean \pm SEM. Asterisks signify significant differences using 1-way ANOVA with Dunnett's multiple comparisons post hoc test. ${ }^{* *} P<0.01,{ }^{*} P<0.05, \mathrm{DF}=4, \mathrm{~F}=7.07$. For all survival curves, significant differences were calculated using log-rank (Mantel-Cox) analysis. PO, postnatal day 0; P21, postnatal day 21; hydral, hydralazine; bical, bicalutamide; spiro, spironolactone. All findings from drug trials are based on analyses using a control group with $n=93$ across all drug tests. FDR-adjusted $P$ values are presented in Supplemental Table 3 .

genesis in a context with intuitive relevance to the human condition and for the use of vascular rupture (as opposed to surrogate biomechanical or histological markers) as the outcome parameter in therapeutic trials. This is especially important given prior work that has failed to demonstrate a reliable correlation between measures of mechanical integrity or histological changes and clinical outcome in either people or mice with vEDS $(11,14-17,22,36)$.

Informatively, in Col3a1 $1^{\mathrm{G} 938 \mathrm{D} /+}$ mouse models, reduction of blood pressure with amlodipine, propranolol, losartan, or atenolol had no therapeutic effect, suggesting that decreasing wall stress on intrinsically fragile arteries does not influence the risk of rupture (Supplemental Figure 4).
The conclusions of one trial suggesting that treatment of vEDS patients with celiprolol delays the onset of vascular complications by reducing mechanical stress on the vascular wall are severely limited by study design, insufficient sample size, and lack of a molecular diagnosis in approximately one-third of patients $(11,13,37)$. A follow-up description of clinical experience with celiprolol in vEDS was insufficient to allow conclusions regarding efficacy or safety given the lack of appropriate controls, randomization, observer blinding, or an intention-to-treat study design $(28,37)$. In our study, celiprolol, while having the predicted effect on pulse rate, accelerated rather than prevented death from aortic dissection in both the severe Col3a1 $1^{\mathrm{G} 938 \mathrm{D} /+}$ and mild Col3a1 ${ }^{\mathrm{G} 209 \mathrm{~S} /+}$ vEDS mouse models (Supplemental Figure 4). 

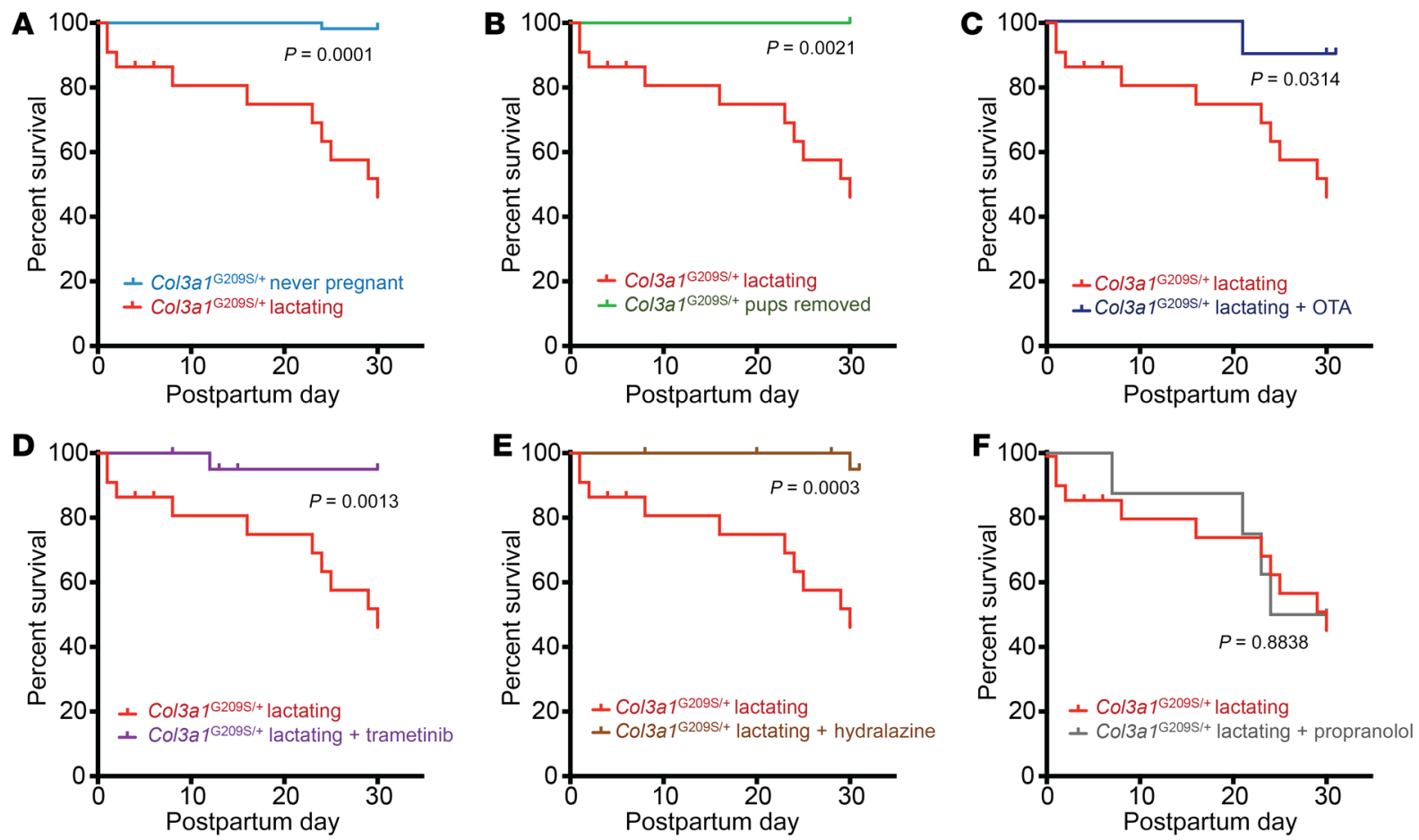

Figure 5. Oxytocin signaling during lactation increases the risk of aortic rupture. (A) Kaplan-Meier survival curve for lactating Col3a ${ }^{62095 /+}$ mice $(n=22)$ compared with never-pregnant female $(n=55)$ Col3a ${ }^{62095 /+}$ mice. (B) Kaplan-Meier survival curve comparing Col3a ${ }^{12095 /+}$ lactating $(n=22)$ mice to Col3a1 $2095 /+$ females with pups removed on the day of delivery thereby preventing lactation and eliminating the lactation-induced prolonged elevation of oxytocin $(n=13)$. (C) Kaplan-Meier survival curve comparing Col3a $1^{\mathrm{C2095} /+}$ lactating mice $(n=22)$ to Col3a $1^{\mathrm{C2} 2095 /+}$ females with oxytocin receptor antagonist (OTA) administered via a continuous subcutaneous infusion pump implanted at the end of the third week of gestation and continued through the 4 weeks

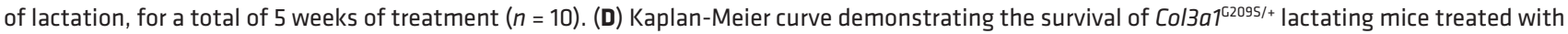
trametinib $(n=21)$, a MEK inhibitor, initiated at the start of the third week of pregnancy and continued through 4 weeks of lactation, in comparison to untreated lactating Col3a1 ${ }^{\mathrm{C2095} /+}$ mice $(n=22)$. (E) Kaplan-Meier curve demonstrating the survival of Col3a $1^{\mathrm{C2} 2095 /+}$ lactating mice treated with hydralazine $(n=23)$ initiated at the start of the third week of pregnancy and continued through 4 weeks of lactation, in comparison to lactating untreated Col3a1c2095/+ mice $(n=22)$. (F) Kaplan-Meier curve demonstrating the survival of Col3a $7^{22095 /+}$ lactating mice treated with propranolol $(n=8)$, initiated at the start of the third week of pregnancy and continued through 4 weeks of lactation, in comparison to lactating untreated Col3a $1^{\mathrm{C2095} /+}$ mice $(n=22)$. For all survival curves, significant differences were calculated using log-rank (Mantel-Cox) analysis, and controls are pooled analyses of $n=22$ mice. FDR-adjusted $P$ values are presented in Supplemental Table 3.

The fact that propranolol, a nonspecific $\beta 1 / \beta 2$ antagonist, and atenolol, a specific $\beta 1$ antagonist, had no impact on survival (Supplemental Figure 4), suggests the deleterious effect of celiprolol may be related to the drug's $\beta 2$ agonism and/or $\alpha 2$ antagonism (38).

ER stress and consequent autophagy due to abnormal collagen secretion has also been proposed as a possible pathogenic mechanism by which alterations in collagen III biosynthesis may lead to altered tissue mechanics and integrity $(16,26)$. Fibroblasts in skin biopsies from individuals with vEDS demonstrate marked rough ER distention (26) and transcriptomic analysis of skin fibroblasts with COL3A1 mutations demonstrated changes in expression of several genes involved in ER homeostasis (39). However, rough ER distention has not been observed in arteries from humans or animal models with vEDS (16). In both Col3a1 $1^{\mathrm{G} 209 \mathrm{~S} /+}$ and Col3a1 ${ }^{\mathrm{G} 938 \mathrm{D} /+}$ mice, we only observed ER distention in the adventitial fibroblasts, but not in endothelial cells or VSMCs, in the descending thoracic aorta (Supplemental Figure 3). We also did not see a signature for ER stress in our transcriptome analysis. In view of these considerations, it appears that neither mere tissue fragility nor ER stressdriven molecular phenotypes explain the high risk of vascular rupture in Col3a1 $1^{\mathrm{G} 209 \mathrm{~S} /+}$ and Col3a1 ${ }^{\mathrm{G} 938 \mathrm{D} /+}$ vEDS mice.
We propose what we believe to be a novel understanding of vEDS pathogenesis, in which the risk of vascular rupture is mediated by excessive signaling through the $\mathrm{PLC} / \mathrm{IP}_{3} / \mathrm{PKC} / \mathrm{ERK}$ axis. This model is supported by unbiased expression profiling of a vulnerable segment of the aortic wall in vEDS mice and by the phenotypic rescue observed upon pharmacologic attenuation of $\mathrm{IP}_{3}$ (hydralazine), PKC $\beta$ (ruboxistaurin), or MEK/ERK (cobimetinib) (Figures 2-4 and Supplemental Figures 5-7), establishing that increased phosphorylation is a cause (and not simply a consequence) of the pathology. Immunoblots confirmed that the status of signaling correlates directly with the risk of aortic rupture and death (Figures 3 and 4). Curiously, although our model puts ERK1/2 downstream of PKC $\beta$, MEK inhibition reduced both ERK1/2 and PKC $\beta$ phosphorylation, suggesting the presence of a yet-unidentified positive feedback loop.

There are multiple mechanisms by which a collagen deficiency could lead to abnormal PLC/IP $/$ / PKC/ERK signaling. Known physiologic activators include receptor tyrosine kinases, integrins, and $\mathrm{G}_{\alpha q}$ GPCRs (40-44). A deficiency of collagen III has been associated with alterations in the level and repertoire of integrin receptors expressed at the cell surface $(45,46)$. Alternatively, vEDS 
A
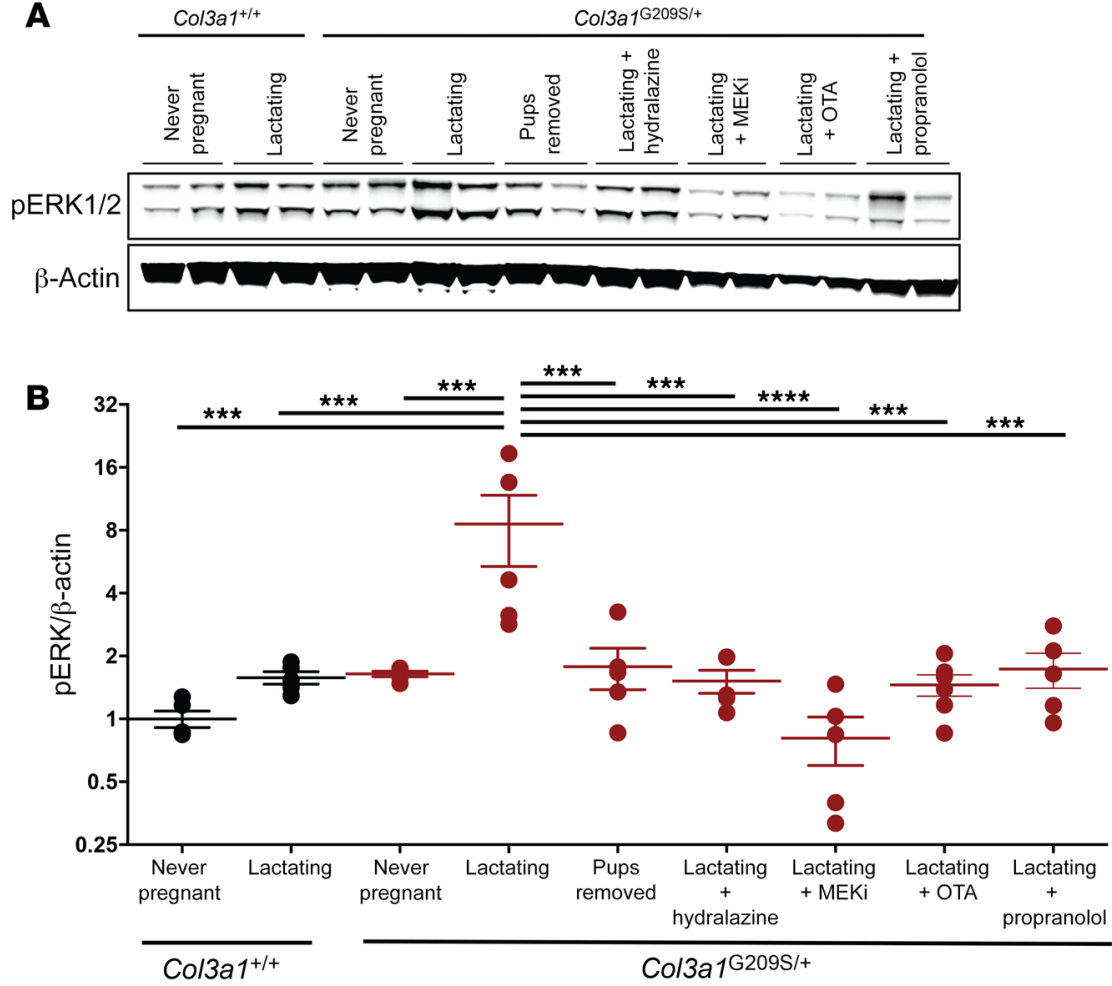

C
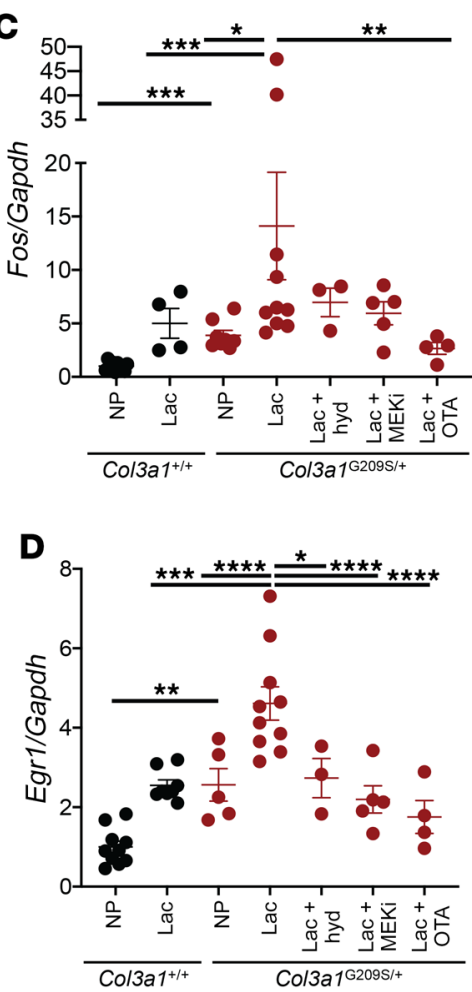

Figure 6. Inhibition of oxytocin signaling decreases ERK activation and ERK target gene expression. (A) Representative Western blot analysis of

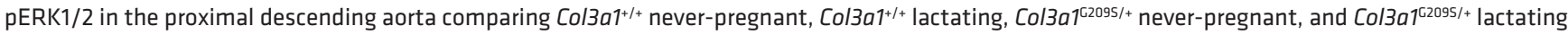
mice, Col3a ${ }^{\mathrm{C2} 2095 /+}$ mice with pups removed thereby preventing lactation, and Col3a ${ }^{\mathrm{C2095} /+}$ mice treated with trametinib (MEKi), oxytocin receptor antagonist (OTA), hydralazine, or propranolol. (B) Quantification of Western blot analysis of pERK1/2 in the proximal descending aorta in Col3a $7^{+/+}$never-pregnant

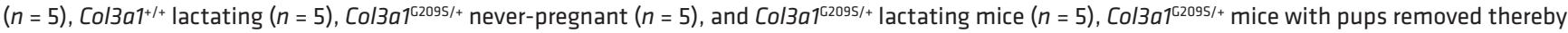
preventing lactation $(n=5)$, and Col3a ${ }^{\mathrm{CL2095} /+}$ mice treated with trametinib $(n=5$, MEKi), oxytocin receptor antagonist $(n=5$, OTA), hydralazine $(n=3)$, or propranolol $(n=5)$. Error bars show mean \pm SEM. Asterisks signify significant differences of log-transformed data using 1-way ANOVA with Dunnett's multiple comparisons post hoc test. ${ }^{* *} P<0.001$. $D F=8, F=8.06$. (C) qPCR analysis of Fos normalized to Capdh in the proximal descending aorta comparing wild-type never-pregnant $(n=6, N P)$, wild-type lactating $(n=4, L A C)$, and Col3a $1^{\mathrm{C2} 2095 /+}$ never-pregnant mice $(n=8, N P)$, Col3a ${ }^{62095 /+}$ lactating mice $(n=10)$, and Col3a $7^{\mathrm{C2095} /+}$ mice treated with trametinib $(n=5, \mathrm{LAC}+$ MEKi), oxytocin receptor antagonist $(n=4, \mathrm{LAC}+$ OTA), or hydralazine $(n=3$, LAC + hyd $)$. Error bars show mean $\pm \mathrm{SEM}$. Asterisks signify significant differences of log-transformed data using 1-way ANOVA with Dunnett's multiple comparisons post hoc test. ${ }^{*} P<0.05,{ }^{* *} P<0.01,{ }^{* *} P<0.001$. DF $=6, F=13.84$. (D) qPCR analysis of Egr1 normalized to Gapdh in the proximal descending aorta comparing

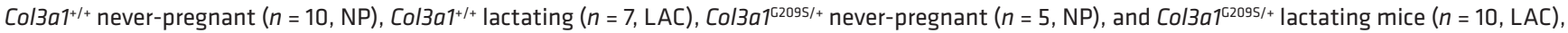
and Col3a $1^{62095 /+}$ mice treated with trametinib $(n=5, \mathrm{LAC}+$ MEKi), oxytocin receptor antagonist $(n=4, \mathrm{LAC}+$ OTA), or hydralazine $(n=3$, LAC + hyd). Error bars show mean \pm SEM. Asterisks signify significant differences using 1-way ANOVA with Dunnett's multiple comparisons post hoc test. ${ }^{*} P<0.05$,

${ }^{* *} P<0.01,{ }^{* *} P<0.001,{ }^{* * * *} P<0.0001 . \mathrm{DF}=6, \mathrm{~F}=14.84$

might be associated with changes in the expression or localization of integrin ligands. While our expression profiling analyses did not reveal suggestive changes in mRNA expression, these possibilities remain incompletely explored. There are no proposed mechanisms by which collagen III deficiency would increase the expression or activity of $\mathrm{G}_{\alpha \mathrm{q}}$ GPCRs, including the angiotensin II, thrombin, and endothelin receptors. None showed enhanced expression in the vEDS descending thoracic aorta. Although others have shown that angiotensin II administration promotes thoracic aortic dissection in Col3a1 haploinsufficient mice (17), angiotensin II receptor blockers (ARBs) did not improve survival in Col3a1 ${ }^{\mathrm{G} 938 \mathrm{D} /+}$ animals, suggesting that angiotensin II receptor signaling is not driving vascular rupture in our model (Supplemental Figure 4). The adhesion GPCR GPR56 uses collagen III as a ligand and is expressed in the aorta, but complete collagen III deficiency phenocopies the polymicrogyria phenotype seen upon loss of function of GPR56, making a gain-of-function in vEDS difficult to reconcile $(41,42,47)$.
Female patients with vEDS are at risk for aortic rupture associated with pregnancy and childbirth. Traditionally, this risk has been ascribed to labor-induced stress, but, as with MFS (32), we noted that aortic rupture generally occurs in the postpartum period (34). Oxytocin signaling plays a key role in aortic rupture, as evidenced by the fact that prevention of lactation, treatment with oxytocin antagonism, or inhibition of signaling pathways activated by oxytocin all increase postpartum survival (Figures 5 and 6). The oxytocin receptor is also a $G_{a q}$ GPCR that signals through the $\mathrm{PLC} / \mathrm{IP}_{3} / \mathrm{PKC} / \mathrm{ERK}$ axis. This deleterious gene-by-environment interaction further supports the hypothesis that overactivation of $\mathrm{PLC} / \mathrm{IP}_{3} / \mathrm{PKC} / \mathrm{ERK}$ signaling promotes vascular rupture in vEDS.

In this work, we identified a role for androgen signaling in vEDS pathogenesis. Mice treated with hydralazine, which inhibits $\mathrm{IP}_{3}$-mediated $\mathrm{PKC} \beta$ activation, had remarkable protection until puberty, at which time male mice experienced significant rates of aortic rupture. An elevated risk of vascular rupture is also seen in 
young men with vEDS commencing toward the end of puberty, but this has not been mechanistically characterized $(2,11,48)$. In our model this elevated risk was rescued by treatment with androgen receptor antagonists and correlated directly with changes in ERK1/2 activation in the aortic wall. It is informative that the vulnerability seen in hydralazine-treated mice at sexual maturity is not observed in mice treated with either PKC $\beta$ or MEK/ERK inhibitors (Figures 3 and 4). Taken together, these data suggest that androgen-mediated enhancement of risk for aortic rupture occurs at a level downstream of $\mathrm{IP}_{3}$ but proximal to $\mathrm{PKC} \beta$ activation, or that androgens activate PKC $\beta$ via an independent (e.g., diacylglycerol) pathway. Female mice treated with hydralazine also showed an increased rate of aortic rupture at sexual maturity, albeit to a lesser extent than that seen in males (Figure 4). This risk was also rescued by treatment with direct androgen receptor antagonists (Figure 4) and so is androgen-mediated, but likely attenuated due to the lower levels of androgens in females (49). Furthermore, the incidence and severity of aneurysm and dissection is greater in males with MFS (50), abdominal aortic aneurysm (51), and other presentations of thoracic aortic aneurysm (52). In this light, androgen signaling may play a broader role in aortic aneurysm and dissection, but the exact mechanism still needs to be elucidated. Prior experience in experimentally induced aneurysm in rodents has suggested that androgen signaling can exacerbate aortic wall inflammation, but little is known about the precise mechanism (51, 53-59). It is notable that in our mouse model of vEDS, the androgen-dependent risk seen at puberty was not associated with overt inflammation.

In anticipation of a reluctance to consider use of potent androgen receptor antagonists in adolescent males with vEDS, we considered the potential use of spironolactone. Spironolactone is a diuretic that reduces adrenal androgen production, is a competitive antagonist of the androgen receptor, and is used clinically to treat hyperandrogenism in the context of skin and hair disorders $(60,61)$. Spironolactone is commonly used in children of both sexes for its diuretic effect. While males can experience mild gynecomastia and females can show menstrual irregularity, these effects are dose- and duration-dependent and are reversible with drug cessation. In our model, the combination of spironolactone and hydralazine in mice of both sexes led to $100 \%$ survival, comparable to the combination of hydralazine with more potent androgen receptor antagonists (Figure 4).

Our results provide the first evidence for a targetable signaling abnormality that contributes to the pathogenesis of vEDS and illustrate the promise of therapeutic strategies aimed at inhibition of the $\mathrm{PLC} / \mathrm{IP}_{3} / \mathrm{PKC} / \mathrm{ERK}$ axis of activation. While therapeutic studies in mice can be difficult to translate to people, these models can assist in the identification of biomarkers of disease risk, progression, and therapeutic responses in patients with vEDS. Additional work will be needed to elucidate the nature of outside-in cellular signaling that initiates with collagen III deficiency, crosstalks with androgen signaling, and culminates in PKC/ERK activation and arterial rupture in vEDS.

\section{Methods}

Generation of Col3a1 knockin mice. To generate Col3a1 knockin mice, we targeted the Col3a1 locus using CRISPR/Cas9 (clustered, regularly interspaced palindromic repeat-CRISPR-associated protein 9). Two single-guide RNA (sgRNA) sequences for each intended mutation (see Supplemental Table 1) were designed to target exon 39 or exon 7 of Col3a1 (NC_000067.6) using a gRNA CRISPR design tool (crispr. mit.edu). The gRNAs were predicted to have negligible off-target effects. The sgRNA was transcribed in vitro. The homology directed repair (HDR) template was purchased as a 4-nmol Ultramer (IDT, see Supplemental Table 1). For the Col3a1 ${ }^{\mathrm{G} 938 \mathrm{D} /+}$ mouse, the sgRNA, Cas9 (TriLink BioTechnologies), and HDR were coinjected into C57BL/6J zygotes (Johns Hopkins University Transgenic Core). For the Col3a $1^{\mathrm{G} 209 \mathrm{~S} / \mathrm{+}}$ mouse, the guide RNA sequences were ligated into the pX330 vector (Addgene, plasmid 42230) according to Cong et al. (23). Briefly, plasmid was linearized with BbsI (R3539S, New England Biolabs) and gRNAs were ligated into the restriction site and verified through Sanger sequencing. In vitro transcription of the cloned gRNAs was performed using MEGAscript T7 Transcription Kit (Life Technologies). The amplicon was purified with a PCR purification kit (Qiagen) and was used as a template for the IVT kit, according to the suggested protocol. All mice were maintained on a C57BL/6J background (The Jackson Laboratory, catalog 000664).

Mutation validation, Sanger sequencing, and genotyping. Bidirectional Sanger DNA sequencing assays were performed using primers designed 100-200 bp from the intended mutation to confirm correct insertion. PCR was performed using a DNA Engine Dyad thermal cycler (Bio-Rad). iProof High Fidelity PCR Master Mix (Bio-Rad) was used in accordance with the manufacturer's instructions for each primer set. Cycle sequencing was performed using the BigDye Terminator v3.1 kit and an ABI 3730xl DNA Analyzer in accordance with the manufacturer's instructions (Life Technologies). Samples were purified using the QIAquick PCR Purification kit (Qiagen). After confirmation of the intended mutation, restriction enzymes were used to detect the presence or absence of the mutation. The G209S mutation leads to the loss of an AvaII cut site and the G938D mutation leads to the gain of a BamHI cut site (AvaII R0153L; BamHI-HF R3136S, New England Biolabs).

All mice found dead were assessed for cause of death by necropsy, noting in particular hemothorax and hemoperitoneum.

Echocardiography. Mice were imaged as previously described (18). Briefly, echocardiograms were performed on awake, unsedated mice using the Visualsonics Vevo 2100 imaging system and a $30 \mathrm{MHz}$ transducer. Three separate measurements of the maximal internal dimension at the aortic root and proximal ascending aorta were made from distinct captured images and averaged. All imaging and measurements were performed by a researcher who was blinded to genotype.

Histology. Mice were euthanized by isoflurane inhalation and the left common iliac artery was transected to allow for drainage. PBS (pH 7.4) and PBS containing 4\% paraformaldehyde (PFA) was flushed through the left ventricle. The heart and thoracic aorta were removed en block and fixed in $4 \%$ PFA overnight at $4^{\circ} \mathrm{C}$. Aortas were submitted for paraffin fixation and longitudinal sections $5-\mu \mathrm{m}$ thick were mounted on glass slides and stained with H\&E, Verhoeff-van Giesen (VVG), Masson's Trichrome, or Picrosirius red (PSR). Slides were imaged at $\times 20$ and $\times 40$ magnification using a Nikon Eclipse E400 microscope. Collagen content was determined by polarized PSR intensity (62) and elastin breaks were counted by a researcher blinded to genotype and treatment arm using only VVG-stained sections where elastin breaks were clearly visualized. 
Electron microscopy. Electron microscopy was performed as previously described (63), focusing on the proximal descending thoracic aortic wall.

RNAseq. RNA was isolated from the proximal descending thoracic aorta of 3 mice for each condition, flushed in PBS, and directly stored into TRIzol (Invitrogen). RNA was extracted according to manufacturer's instructions and purified using the PureLink RNA Mini Kit (Invitrogen). Library prep was performed using TruSeq Stranded Total RNA with Ribo-Zero (Illumina). Sequencing was run on an Illumina HiSeq2500 using standard protocols.

Bioinformatics. Illumina's CASAVA 1.8.4 was used to convert BCL files to FASTQ files. Default parameters were used. rsem-1.3.0 was used for running the alignments as well as generating gene and transcript expression levels. The data were aligned to "mm10" reference genome. EBseq was used for differential expression analysis and default parameters were used (64). The networks and upstream regulator analyses were generated through the use of IPA (Qiagen, https://www. qiagenbioinformatics.com/products/ingenuity-pathway-analysis).

Western blot. Descending thoracic aortas (distal to the left subclavian branch and proximal to the diaphragm) from mice that did not die from aortic rupture and did not have any overt pathology at the time of planned sacrifice (at 2 months of age for all samples unless otherwise stated) were harvested, snap frozen in liquid nitrogen, and stored at $-80^{\circ} \mathrm{C}$ until processed. Protein was extracted using an automatic bead homogenizer in conjunction with a Protein Extraction Kit (Full Moon Biosystems). All protein lysis buffers contained both PhosSTOP and cOmplete, Mini, EDTA-free Protease Inhibitor Cocktail (Roche). Western blotting was performed using LI-COR buffer and speciesappropriate secondary antibodies conjugated to IR-dye700 or IRdye800 (LI-COR Biosciences), according to the manufacturer's guidelines and analyzed using LI-COR Odyssey. The following primary antibodies were used: anti- $\beta$-Actin (8H10D10) (Cell Signaling Technology, catalog 3700), anti-phospho ERK1/2 (Cell Signaling Technology, catalog 4370), anti-PKC $\beta$ (phospho S660) (Abcam, catalog 75837).

pERK and pPKC amounts were normalized to $\beta$-actin as opposed to total ERK or total PKC for a variety of practical reasons. First, it is the amount of phosphorylated protein, and not the ratio of phosphorylated to unphosphorylated protein, that drives cellular responses. Second, we are unaware of any pathophysiologic context where the amount of unphosphorylated protein is limiting (i.e., always in excess). Third, we and others have shown that the heterogeneous nature of cells that are accumulated, recruited, and/or expanded in vascular lesions generates extreme variability and potential artifact when phosphorylated-to-unphosphorylated ratios are used $(65,66)$. This is true because many of the resident and recruited cell types in the diseased aorta express high levels of the unphosphorylated protein but are not involved in the pathologic process leading to protein activation (e.g., inflammatory cells, adventitial fibroblasts). The abundance of these cells can lead to masking of the activation signal that is only present in a small subset of critical cells.

Gene expression. Aortas were dissected as described above, flushed in PBS, and directly stored in TRIzol (Invitrogen). RNA was extracted according to the manufacturer's instructions and purified with RNAeasy mini columns (Qiagen). Complementary DNA (cDNA) was generated using TaqMan High Capacity cDNA Reverse Transcription reagents (Applied Biosystems) and qPCR was performed in triplicate with TaqMan Universal PCR Master Mix (Applied Biosystems).
The following TaqMan probes were used: Mm00487425_m1 (Fos), Mm00656724_m1 (Egr1), Mm00442688_m1 (Ar), Mm99999915_g1 (Gapdh), Mm00446968_m1 (Hprt). Relative quantification for each transcript was obtained by normalizing against Gapdh or Hprt (as indicated in each figure) transcript abundance according to the formula $2^{(-\mathrm{Ct})} / 2^{(-\mathrm{Ct} \mathrm{Gapdh})}$. All expression levels were normalized to untreated wildtype control expression levels.

Delivery of medication. For drug trials in the Col3a $1^{\mathrm{G} 938 \mathrm{D} /+}$ mice, mice were initiated on medication at weaning and continued until 2 months of age. Cobimetinib (GDC-0973/RO551404, Active Biochem) was dissolved in drinking water and filtered to reach a final concentration of $0.02 \mathrm{~g} / \mathrm{L}$, giving an estimated dose of $2 \mathrm{mg} / \mathrm{kg} / \mathrm{day}$. Ruboxistaurin (LY333531 HCl, Selleck Chemicals) was mixed with powdered food (LabDiet) to give a concentration of $0.1 \mathrm{mg} / \mathrm{g}$, giving an estimated dose of $8 \mathrm{mg} / \mathrm{kg} /$ day. Hydralazine (Exelan) is known to be safe in pregnancy and is found in breastmilk, so was initiated at birth and dissolved in drinking water and filtered to reach a final concentration of $0.32 \mathrm{~g} / \mathrm{L}$, giving an estimated daily dose of $32 \mathrm{mg} /$ $\mathrm{kg}$ /day. Bicalutamide (Major Pharmaceuticals) was mixed with powdered food to give a concentration of $0.625 \mathrm{mg} / \mathrm{g}$, giving an estimated dose of $50 \mathrm{mg} / \mathrm{kg} /$ day. Losartan (Merck) was dissolved in drinking water and filtered to reach a final concentration of $0.6 \mathrm{~g} / \mathrm{L}$, giving an estimated dose of $60 \mathrm{mg} / \mathrm{kg} /$ day. Celiprolol (Pfizer) was dissolved in drinking water and filtered to reach a final concentration of $2 \mathrm{~g} / \mathrm{L}$, giving an estimated dose of $200 \mathrm{mg} / \mathrm{kg} /$ day. Propranolol (Qualitest) was dissolved in the drinking water and filtered to reach a final concentration of $0.8 \mathrm{~g} / \mathrm{L}$, giving an estimated dose of $80 \mathrm{mg} / \mathrm{kg} / \mathrm{day}$. Atenolol (Teva) was dissolved in the drinking water and filtered to reach a final concentration of $1.2 \mathrm{~g} / \mathrm{L}$, giving an estimated dose of $120 \mathrm{mg} / \mathrm{kg} / \mathrm{day}$. Amlodipine besylate (Zygenerics) was dissolved in drinking water and filtered to reach a final concentration of $0.12 \mathrm{~g} / \mathrm{L}$, giving an estimated dose of $12 \mathrm{mg} / \mathrm{kg} /$ day. Spironolactone (Sky) was mixed with powdered food (LabDiet) to give a concentration of $1.25 \mathrm{mg} / \mathrm{g}$, giving an estimated dose of $100 \mathrm{mg} / \mathrm{kg} /$ day. Wild-type mice treated at these doses did not show any deleterious effects on survival (Supplemental Table 4).

For the drug trials in pregnancy, mice were initiated on medication at the third week of gestation and continued for 1 month postpartum. The selective oxytocin antagonist (des Gly-NH2,d(CH2)5[DTyr2,Thr4]OVT) was synthesized in the laboratory of Maurice Manning (University of Toledo) and was delivered in PBS to reach a final dose of $1 \mu \mathrm{g} / \mathrm{kg} / \mathrm{hr}$ when delivered via continuous infusion in a mini Alzet pump implanted subcutaneously between the scapulae. Propranolol (Qualitest) was dissolved in the drinking water and filtered to reach a final concentration of $0.8 \mathrm{~g} / \mathrm{L}$, giving an estimated dose of $80 \mathrm{mg} / \mathrm{kg} /$ day. Hydralazine (Exelan) was dissolved in drinking water and filtered to reach a final concentration of $0.16 \mathrm{~g} / \mathrm{L}$, giving an estimated daily dose of $16 \mathrm{mg} / \mathrm{kg} /$ day. Trametinib (GSK) was dissolved in PBS with 10\% DMSO and mice were treated once a day by oral gavage, giving an estimated daily dose of $1 \mathrm{mg} / \mathrm{kg} /$ day. Placebotreated animals received regular drinking water.

Blood pressure analysis. Blood pressures were measured by tail cuff plethysmography 1 week prior to completion of a study. To measure and record blood pressures, we utilized the BP-2000 Blood Pressure Analysis system. This method utilizes variations in the amount of light transmitted through the tail as the basic signal that is analyzed to determine the blood pressure and pulse rate. After the software determines the pulse rate, it inflates the occlusion cuff and records diastolic 
pressure when the wave form starts to decrease and systolic pressure when the waveform remains at a steady value. If either measurement is unclear to the software, it is not recorded. Mice were habituated to the system for 3 days prior to collection, in which 10-15 measurements were obtained and averaged. Sex-matched controls were measured at the same time as experimental mice to account for day-to-day and sex-dependent variability in mouse blood pressure.

Statistics. All data points are presented for quantitative data, with an overlay of the mean with SEM. All statistical analysis was performed using GraphPad Prism 8 unless otherwise noted. For data that did not pass Shapiro-Wilk normality tests, Kruskal-Wallis (nonparametric) tests were performed to evaluate significance between groups using Dunn's multiple comparison test with a $P$ value of less than 0.05 considered statistically significant. For data that did pass normality, 2-way or 1-way ANOVA was used with multiple comparisons, as noted in each legend.

For single comparisons, if the Shapiro-Wilk normality test was passed, then 2-tailed unpaired $t$ tests were performed. If Shapiro-Wilk normality test did not pass, then Mann-Whitney nonparametric tests were performed. Kaplan-Meier survival curves were compared using a log-rank (Mantel-Cox) test. Mice were censored only if unrelated to the outcome, such as for planned biochemical or histologic analysis or if the authors were directed to euthanize them by animal care staff, for malocclusion, fight wounds, or genital prolapse.

By design, each treatment trial included contemporaneous control (untreated) mice. The performance of untreated mice remained constant for the full duration of this study (Supplemental Figure 10), allowing pooling of controls to improve statistical power, as per our usual practice (32). All findings from our drug trials are based on analyses using a universal control group with $n=93$ across all drug tests that started at P21. For drug trials that started at PO, a pooled control group followed from day PO with $n=51$ was used. All findings from our pregnancy trials are based on analyses of $n=22$ mice. All $P$ values calculated by log-rank comparisons (using Prism) are reported as unadjusted $P$ values, given the interdependence of many of the drug mechanisms. We also report FDR-adjusted $P$ values for the number of comparisons across the entire study (calculated using R [ref. 67]) (Supplemental Table 3).

For all treatment trials, untreated mice were followed for the same duration (i.e., if a treatment trial did not initiate until age 21 days, control cohorts were followed starting at age 21 days). For treatment trials that initiated at birth (PO), separate cohorts of mice were also followed starting from birth (PO).
Study approval. All mice were cared for under strict adherence to the Animal Care and Use Committee of the Johns Hopkins University School of Medicine.

Data availability. RNA sequencing data are available from the Sequence Read Archive (SRA) under accession PRJNA532935. All other data associated with this study are present in the paper or Supplemental Material.

\section{Author contributions}

CJB and HCD were responsible for all aspects of study design, data interpretation, and preparation of the manuscript. CJB, ZB, and GR were responsible for mouse husbandry and medication dosing. CJB, JFCG, GR, and MRH created and phenotyped mouse models. ECD performed electron microscopy studies. CJB interpreted echocardiograms. CJB and KB performed data analysis. CJB, EGM, and HCD wrote the manuscript with contributions from all authors.

\section{Acknowledgments}

We thank the staff of the Next Generation Sequencing Center, Research Animal Resources, and the Transgenic Core Laboratory at Johns Hopkins University School of Medicine for their assistance with various aspects of this project. We thank Jennifer Habashi and Djahida Bedja (Johns Hopkins University) for their assistance with echocardiograms and Maurice Manning (University of Toledo) for providing the oxytocin receptor antagonist and guidance regarding its use in animal studies. Supported by grants from the NIH (AR41135 to HCD; GM007309 to CJB), the Howard Hughes Medical Institute (to HCD), the Marfan Foundation (to HCD), the Ehlers Danlos Syndrome Network C.A.R.E.S. Foundation (to HCD), EDS Today (to HCD), the Daskal Family Foundation (to HCD), the Alison Aldredge Family Foundation (to HCD), the DEFY Foundation (to HCD), the Natural Sciences and Engineering Research Council of Canada (to ECD), the Fulbright-Conicyt Scholarship (to JFCG), and Fondecyt Grant (11170353 to JFCG).

Address correspondence to: Harry C. Dietz, Johns Hopkins University School of Medicine, Miller Research Building Room 539, 733 North Broadway, Baltimore, Maryland 21205, USA. Phone: 410.614.0701; Email: hdietz@jhmi.edu.

JFCG's present address is: Centro de Genética y Genómica, ICIM, Facultad de Medicina Clínica Alemana Universidad del Desarrollo, Santiago, Chile.
1. Pepin M, Schwarze U, Superti-Furga A, Byers PH. Clinical and genetic features of Ehlers-Danlos syndrome type IV, the vascular type. $N$ Engl J Med. 2000;342(10):673-680.

2. Pepin MG, Schwarze U, Rice KM, Liu M, Leistritz $\mathrm{D}$, Byers $\mathrm{PH}$. Survival is affected by mutation type and molecular mechanism in vascular Ehlers-Danlos syndrome (EDS type IV). Genet Med. 2014;16(12):881-888.

3. Shalhub S, et al. Molecular diagnosis in vascular Ehlers-Danlos syndrome predicts pattern of arterial involvement and outcomes. J Vasc Surg. 2014;60(1):160-169.

4. Smith LB, et al. Haploinsufficiency of the murine
Col3a1 locus causes aortic dissection: a novel model of the vascular type of Ehlers-Danlos syndrome. Cardiovasc Res. 2011;90(1):182-190.

5. Wilson NK, Gould RA, Gallo MacFarlane E, Mibava Leducq Consortium. Pathophysiology of aortic aneurysm: insights from human genetics and mouse models. Pharmacogenomics. 2016;17(18):2071-2080.

6. Holm TM, et al. Noncanonical TGF $\beta$ signaling contributes to aortic aneurysm progression in Marfan syndrome mice. Science. 2011;332(6027):358-361.

7. Dale M, et al. Premature aortic smooth muscle cell differentiation contributes to matrix dys- regulation in Marfan syndrome. PLOS ONE. 2017;12(10):e0186603.

8. Gallo EM, et al. Angiotensin II-dependent TGF- $\beta$ signaling contributes to Loeys-Dietz syndrome vascular pathogenesis. J Clin Invest. 2014;124(1):448-460.

9. Leistritz DF, Pepin MG, Schwarze U, Byers PH. COL3A1 haploinsufficiency results in a variety of Ehlers-Danlos syndrome type IV with delayed onset of complications and longer life expectancy. Genet Med. 2011;13(8):717-722.

10. Cortini F, et al. A new COL3A1 mutation in Ehlers-Danlos syndrome vascular type with different phenotypes in the same family. Vasc 
Endovascular Surg. 2017;51(3):141-145.

11. Byers PH, et al. Diagnosis, natural history, and management in vascular Ehlers-Danlos syndrome. Am JMed Genet C Semin Med Genet. 2017;175(1):40-47.

12. Kuivaniemi H, Tromp G. Type III collagen (COL3A1): Gene and protein structure, tissue distribution, and associated diseases. Gene. 2019;707:151-171.

13. Ong KT, et al. Effect of celiprolol on prevention of cardiovascular events in vascular Ehlers-Danlos syndrome: a prospective randomised, open, blinded-endpoints trial. Lancet. 2010;376(9751):1476-1484.

14. Briest W, Cooper TK, Tae HJ, Krawczyk M, McDonnell NB, Talan MI. Doxycycline ameliorates the susceptibility to aortic lesions in a mouse model for the vascular type of Ehlers-Danlos syndrome. J Pharmacol Exp Ther. 2011;337(3):621-627.

15. Cooper TK, et al. The haploinsufficient Col3a1 mouse as a model for vascular Ehlers-Danlos syndrome. Vet Pathol. 2010;47(6):1028-1039.

16. D'hondt S, et al. Type III collagen affects dermal and vascular collagen fibrillogenesis and tissue integrity in a mutant Col3a1 transgenic mouse model. Matrix Biol. 2018;70:72-83.

17. Faugeroux J, et al. Angiotensin II promotes thoracic aortic dissections and ruptures in Col3a1 haploinsufficient mice. Hypertension. 2013;62(1):203-208.

18. Habashi JP, et al. Angiotensin II type 2 receptor signaling attenuates aortic aneurysm in mice through ERK antagonism. Science. 2011;332(6027):361-365.

19. Doyle JJ, et al. A deleterious gene-by-environment interaction imposed by calcium channel blockers in Marfan syndrome. Elife. 2015;4:e08648.

20. Habashi JP, et al. Losartan, an AT1 antagonist, prevents aortic aneurysm in a mouse model of Marfan syndrome. Science. 2006;312(5770):117-121.

21. Liu X, Wu H, Byrne M, Krane S, Jaenisch R. Type III collagen is crucial for collagen I fibrillogenesis and for normal cardiovascular development. Proc Natl Acad Sci USA. 1997;94(5):1852-1856.

22. Dubacher N, et al. Celiprolol but not losartan improves the biomechanical integrity of the aorta in a mouse model of vascular Ehlers-Danlos syndrome [Published online ahead of print April 8, 2019]. Cardiovasc Res. https://doi.org/10.1093/ $\mathrm{cvr} / \mathrm{cvz} 095$.

23. Cong L, et al. Multiplex genome engineering using CRISPR/Cas systems. Science. 2013;339(6121):819-823.

24. Mizuno K, Boudko S, Engel J, Bächinger HP. Vascular Ehlers-Danlos syndrome mutations in type III collagen differently stall the triple helical folding. J Biol Chem. 2013;288(26):19166-19176.

25. Korneva A, Zilberberg L, Rifkin DB, Humphrey JD, Bellini C. Absence of LTBP-3 attenuates the aneurysmal phenotype but not spinal effects on the aorta in Marfan syndrome. Biomech Model Mechanobiol. 2019;18(1):261-273.

26. Smith LT, Schwarze U, Goldstein J, Byers PH. Mutations in the COL3A1 gene result in the Ehlers-Danlos syndrome type IV and alterations in the size and distribution of the major collagen fibrils of the dermis. JInvest Dermatol.
1997;108(3):241-247.

27. MacFarlane EG, et al. Lineage-specific events underlie aortic root aneurysm pathogenesis in Loeys-Dietz syndrome. J Clin Invest. 2019;129(2):659-675.

28. Frank M, et al. Vascular Ehlers-Danlos syndrome: long-term observational study. JAm Coll Cardiol. 2019;73(15):1948-1957.

29. PKC-DRS2 Group, et al. Effect of ruboxistaurin on visual loss in patients with diabetic retinopathy. Ophthalmology. 2006;113(12):2221-2230.

30. Larkin J, et al. Combined vemurafenib and cobimetinib in BRAF-mutated melanoma. $N$ Engl J Med. 2014;371(20):1867-1876.

31. Gurney AM, Allam M. Inhibition of calcium release from the sarcoplasmic reticulum of rabbit aorta by hydralazine. Br J Pharmacol. 1995;114(1):238-244.

32. Rudd NL, Nimrod C, Holbrook KA, Byers PH. Pregnancy complications in type IV Ehlers-Danlos syndrome. Lancet. 1983;1(8314-5):50-53.

33. Murray ML, Pepin M, Peterson S, Byers PH. Pregnancy-related deaths and complications in women with vascular Ehlers-Danlos syndrome. Genet Med. 2014;16(12):874-880.

34. Habashi JP, et al. Oxytocin antagonism prevents pregnancy-associated aortic dissection in a mouse model of Marfan syndrome. Sci Transl Med. 2019;11(490):eaat4822.

35. Manning M, et al. Oxytocin and vasopressin agonists and antagonists as research tools and potential therapeutics. J Neuroendocrinol. 2012;24(4):609-628.

36. Boutouyrie P, Germain DP, Fiessinger JN, Laloux $\mathrm{B}$, Perdu J, Laurent S. Increased carotid wall stress in vascular Ehlers-Danlos syndrome. Circulation. 2004;109(12):1530-1535.

37. De Backer J, De Backer T. Vascular EhlersDanlos syndrome management: the Paris way, a step forward on a long road. J Am Coll Cardiol. 2019;73(15):1958-1960.

38. Nawarskas JJ, Cheng-Lai A, Frishman WH. Celiprolol: a unique selective adrenoceptor modulator. Cardiol Rev. 2017;25(5):247-253.

39. Chiarelli N, Carini G, Zoppi N, Ritelli M, Colombi M. Transcriptome analysis of skin fibroblasts with dominant negative COL3A1 mutations provides molecular insights into the etiopathology of vascular Ehlers-Danlos syndrome. PLOS ONE. 2018;13(1):e0191220.

40. Judge DP, et al. Evidence for a critical contribution of haploinsufficiency in the complex pathogenesis of Marfan syndrome. J Clin Invest. 2004;114(2):172-181.

41. Luo R, et al. Mechanism for adhesion $G$ protein-coupled receptor GPR56-mediated RhoA activation induced by collagen III stimulation. PLOS ONE. 2014;9(6):e100043.

42. Luo R, Jin Z, Deng Y, Strokes N, Piao X. Diseaseassociated mutations prevent GPR56-collagen III interaction. PLOS ONE. 2012;7(1):e29818.

43. Luttrell LM. Transmembrane signaling by $\mathrm{G}$ protein-coupled receptors. In: Ali $\mathrm{H}$, Bodduluri $\mathrm{H}$, eds. Transmembrane Signaling Protocols. Totowa, New Jersey, USA: Humana Press; 2006:1-50.

44. Natarajan K, Berk BC. Crosstalk coregulation mechanisms of $G$ protein-coupled receptors and receptor tyrosine kinases. In: Ali H, Bodduluri H, eds. Transmembrane Signaling Protocols. Totowa, New Jersey, USA: Humana Press; 2006:51-78.

45. Zoppi N, Ritelli M, Colombi M. Type III and V collagens modulate the expression and assembly of $\operatorname{EDA}(+)$ fibronectin in the extracellular matrix of defective Ehlers-Danlos syndrome fibroblasts. Biochim Biophys Acta. 2012;1820(10):1576-1587.

46. Zoppi N, Gardella R, De Paepe A, Barlati S, Colombi M. Human fibroblasts with mutations in COL5A1 and COL3A1 genes do not organize collagens and fibronectin in the extracellular matrix, down-regulate alpha2beta1 integrin, and recruit alphavbeta3 Instead of alpha5beta1 integrin. J Biol Chem. 2004;279(18):18157-18168.

47. Vandervore $\mathrm{L}$, et al. Bi-allelic variants in COL3A1 encoding the ligand to GPR56 are associated with cobblestone-like cortical malformation, white matter changes and cerebellar cysts. JMed Genet. 2017;54(6):432-440.

48. Byers PH. Vascular Ehlers-Danlos Syndrome. Seattle, Washington, USA: GeneReviews, University of Washington; 1993.

49. Burger HG. Androgen production in women. Fertil Steril. 2002;77(Suppl 4):S3-S5.

50. Roman MJ, et al. Associations of age and sex with Marfan phenotype: the National Heart, Lung, and Blood Institute GenTAC (Genetically Triggered Thoracic Aortic Aneurysms and Cardiovascular Conditions) Registry. Circ Cardiovasc Genet. 2017;10(3):e001647.

51. Henriques T, Zhang X, Yiannikouris FB, Daugherty A, Cassis LA. Androgen increases AT1a receptor expression in abdominal aortas to promote angiotensin II-induced AAAs in apolipoprotein E-deficient mice. Arterioscler Thromb Vasc Biol. 2008;28(7):1251-1256.

52. Losenno KL, Goodman RL, Chu MW. Bicuspid aortic valve disease and ascending aortic aneurysms: gaps in knowledge. Cardiol Res Pract. 2012;2012:145202.

53. Henriques TA, Huang J, D'Souza SS, Daugherty A, Cassis LA. Orchidectomy, but not ovariectomy, regulates angiotensin II-induced vascular diseases in apolipoprotein E-deficient mice. Endocrinology. 2004;145(8):3866-3872.

54. Alsiraj $\mathrm{Y}$, et al. Female mice with an XY sex chromosome complement develop severe angiotensin II-induced abdominal aortic aneurysms. Circulation. 2017;135(4):379-391.

55. Alsiraj Y, Thatcher SE, Blalock E, Fleenor B, Daugherty A, Cassis LA. Sex chromosome complement defines diffuse versus focal angiotensin II-induced aortic pathology. Arterioscler Thromb Vasc Biol. 2018;38(1):143-153.

56. Huang CK, et al. Androgen receptor promotes abdominal aortic aneurysm development via modulating inflammatory interleukin- $1 \alpha$ and transforming growth factor- $\beta 1$ expression. Hypertension. 2015;66(4):881-891.

57. Davis JP, et al. Pharmacologic blockade and genetic deletion of androgen receptor attenuates aortic aneurysm formation. J Vasc Surg. 2016;63(6):1602-1612.e2.

58. Arnold AP, Cassis LA, Eghbali M, Reue K, Sandberg K. Sex hormones and sex chromosomes cause sex differences in the development of cardiovascular diseases. Arterioscler Thromb Vasc Biol. 2017;37(5):746-756. 
59. Zhang X, Thatcher S, Wu C, Daugherty A, Cassis LA. Castration of male mice prevents the progression of established angiotensin IIinduced abdominal aortic aneurysms. J Vasc Surg. 2015;61(3):767-776.

60. Levy LL, Emer JJ. Female pattern alopecia: current perspectives. Int $J$ Womens Health. 2013;5:541-556.

61. Muhlemann MF, Carter GD, Cream JJ, Wise P. Oral spironolactone: an effective treatment for acne vulgaris in women. Br J Dermatol. 1986;115(2):227-232.
62. Vogel B, Siebert H, Hofmann U, Frantz S. Determination of collagen content within picrosirius red stained paraffin-embedded tissue sections using fluorescence microscopy. MethodsX. 2015;2:124-134.

63. Davis EC. Smooth muscle cell to elastic lamina connections in developing mouse aorta. Role in aortic medial organization. Lab Invest. 1993;68(1):89-99.

64. Leng N, et al. EBSeq: an empirical Bayes hierarchical model for inference in RNA-seq experiments. Bioinformatics. 2013;29(8):1035-1043.
65. Daugherty A, Rateri DL, Charo IF, Owens AP, Howatt DA, Cassis LA. Angiotensin II infusion promotes ascending aortic aneurysms: attenuation by CCR2 deficiency in apoE-/- mice. Clin Sci. 2010;118(11):681-689.

66. Anidjar S, Dobrin PB, Eichorst M, Graham GP, Chejfec G. Correlation of inflammatory infiltrate with the enlargement of experimental aortic aneurysms. J Vasc Surg. 1992;16(2):139-147.

67. R Core Team. R: A language and environment for statistical computing. https://www.r-project.org. Accessed 12 November, 2019. 